\title{
Persuading Strategic Voters
}

Citation for published version (APA):

Kerman, T., Herings, P. J-J., \& Karos, D. (2020). Persuading Strategic Voters. Maastricht University, Graduate School of Business and Economics. GSBE Research Memoranda No. 004 https://doi.org/10.26481/umagsb.2020004

Document status and date:

Published: 20/02/2020

DOI:

10.26481/umagsb.2020004

Document Version:

Publisher's PDF, also known as Version of record

\section{Please check the document version of this publication:}

- A submitted manuscript is the version of the article upon submission and before peer-review. There can be important differences between the submitted version and the official published version of record.

People interested in the research are advised to contact the author for the final version of the publication, or visit the DOI to the publisher's website.

- The final author version and the galley proof are versions of the publication after peer review.

- The final published version features the final layout of the paper including the volume, issue and page numbers.

Link to publication

\footnotetext{
General rights rights.

- You may freely distribute the URL identifying the publication in the public portal. please follow below link for the End User Agreement:

www.umlib.nl/taverne-license

Take down policy

If you believe that this document breaches copyright please contact us at:

repository@maastrichtuniversity.nl

providing details and we will investigate your claim.
}

Copyright and moral rights for the publications made accessible in the public portal are retained by the authors and/or other copyright owners and it is a condition of accessing publications that users recognise and abide by the legal requirements associated with these

- Users may download and print one copy of any publication from the public portal for the purpose of private study or research.

- You may not further distribute the material or use it for any profit-making activity or commercial gain

If the publication is distributed under the terms of Article $25 \mathrm{fa}$ of the Dutch Copyright Act, indicated by the "Taverne" license above, 


\section{Maastricht University}

Toygar Kerman,

P. Jean-Jacques Herings,

Dominik Karos

Persuading Strategic Voters

RM/20/004

\section{GSBE}

Maastricht University School of Business and Economics

Graduate School of Business and Economics

\section{P.O Box 616}

NL- 6200 MD Maastricht

The Netherlands 


\title{
Persuading Strategic Voters
}

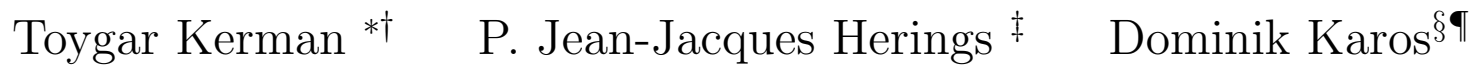 \\ January 16, 2020
}

\begin{abstract}
A Sender wants to persuade multiple Receivers with homogeneous preferences and a common belief about the state of the world to vote in favor of a proposal. Prior to the vote Sender commits to a communication strategy which sends private, potentially correlated, signals to Receivers that are contingent on the true state of the world. While Sender benefits from using private messages rather than public communication if Receivers vote sincerely, under the optimal communication strategy, sincere voting is not in any Receiver's best interest. If the proposal does not require unanimous agreement, Sender's optimal communication strategy after which sincere voting indeed constitutes a Bayes-Nash equilibrium is such that no voter is ever pivotal.
\end{abstract}

Keywords: Bayesian Persuasion, Strategic Voting, Swing Voter's Curse.

JEL codes: C72, D72, D82, D83.

\section{Introduction}

Suppose a country is holding a referendum in order to decide whether or not to adopt a certain policy. Then, typically, government has a strict preference for either the proposal or the status quo. For instance, in the run up to the Brexit referendum, a majority of the cabinet campaigned against leaving the European Union (that is, in favor of the status quo), ${ }^{1}$ whereas in the 2017 Turkish constitutional referendum government campaigned for replacing the existing parliamentary system with a presidential system (that is, in favor of the proposal). Either way, the aim of a political campaign run by government is to increase the probability that their preferred outcome obtains a majority of the votes, that is, to persuade the electorate.

\footnotetext{
*corresponding author

†t.kerman@maastrichtuniversity.nl

${ }^{\ddagger}$ p.herings@maastrichtuniversity.nl

$\S$ The author gratefully acknowledges funding by the ERC, Project Number 747614 .

『d.karos@maastrichtuniversity.nl

${ }^{1}$ https://www.bbc.com/news/uk-politics-eu-referendum-35616946
} 


\subsection{Voting Games}

In a voting game, all voters decide between voting for or against a proposal. The proposal is being implemented if at least $k$ out of $n$ voters vote for it. Voters' utility is determined by the unknown state of the world and the outcome that is being implemented. Throughout the paper we assume that all voters are identical: they have the same prior and their preferences over outcomes are aligned. ${ }^{2}$

The literature mainly distinguishes two forms of voter behavior: sincere voting and strategic voting. ${ }^{3}$ Roughly speaking, a sincere voter operates under the assumption that his vote is decisive, i.e., that his vote decides the election. Hence, he votes for whichever alternative he prefers the most (given his belief) and disregards the behavior of any other voters. A strategic voter, however, takes into account the voting behavior of others and may, hence, vote for an alternative which does not reflect his true preference: doing so might implement an alternative he prefers over the alternative that would have been implemented, had he voted sincerely. ${ }^{4}$

If voters act strategically, a myriad of trivial (and unappealing) equilibria might emerge: for instance, in a simple majority game all voters voting for their least preferred option is an equilibrium as no voter can affect the outcome by changing their vote. Under sincere voting, on the other hand, equilibrium has no meaning as voters ignore the behavior of others. In this paper we will combine the best of both worlds and focus on situations in which sincere voting is an equilibrium. That is, it is optimal for each Receiver to vote sincerely provided that all other voters vote sincerely. This allows us, in particular, to eliminate any trivial equilibria. We illustrate this constraint, which is new to the literature on voter persuasion, with an example.

\subsection{An Illustrating Example}

Consider a government (Sender) who proposes to ban cars on certain days of the year. For simplicity, assume that changing the status quo requires the approval of two out of three committee members (Receivers). Committee members are not certain whether the proposal will benefit $(B)$ or harm $(H)$ society and vote for the proposal if they think that the chance of $B$ is at least $50 \%$. Government wants the proposal to pass independently

\footnotetext{
${ }^{2}$ No idiosyncraticity is one difference with Alonso and Câmara (2016), which employs heteregeneous Receivers and exploits this heterogeneity by targeting different winning coalitions. Chan et al. (2019) consider heterogeneity by assuming voters have different costs to acquire information. For another paper which considers costly information acquisition, see Matyskova (2018).

${ }^{3}$ Spenkuch (2018) argues that evidence suggests that voters cannot be neatly categorized into sincere and strategic "types". However, we stick to the general convention in our model.

${ }^{4}$ Wang (2013) considers sincere voting under public persuasion and strategic voting under private persuasion with independent signals. In Alonso and Câmara (2016) agents have the same beliefs about the state, so there is no need to consider strategic voting.
} 
of its benefit or harm and, hence, wants to persuade voters to approve the proposal. ${ }^{5}$ Government and committee members have a common prior belief that the ban will be beneficial with $30 \%$ probability. ${ }^{6}$ If committee members vote sincerely, then, without intervention, no committee member will vote in favor of the ban and the ban will not be implemented. In particular, this is an equilibrium as no voter could change the outcome by unilaterally changing their vote.

Fully informative research Supose that prior to the vote, government attempts to influence the committee by conducting research and truthfully sharing the results with the committee members. Government can, for example, ask political scientists and economists to report their findings on the possible implications of implementing the ban. ${ }^{7}$ Given the common prior, there is a $30 \%$ chance that this research will prove the ban on cars to be beneficial. Hence, by conducting this research, government can increase the ex ante probability of implementing the ban to $30 \%$.

Persuasion with public signals Government can, however, improve upon this by conducting a partially informative research whose results will be shared publicly. ${ }^{8}$ This could be done, for example, by hiring a partisan economist who does a research which only points out the benefits of implementing the ban. Let government's research be formalized by distributions $\pi(\cdot \mid B)$ and $\pi(\cdot \mid H)$ on some set of signals. Let $\mathbf{b}=(b, b, b)$ and $\mathbf{h}=(h, h, h)$ be the signals in which all members receive a message that recommends to vote either for or, respectively, against the ban. Consider the following distribution. ${ }^{9}$

\begin{tabular}{c|cc}
$\pi^{* \mathrm{p}}$ & $B$ & $H$ \\
\hline $\mathbf{b}$ & 1 & $\frac{3}{7}$ \\
$\mathbf{h}$ & 0 & $\frac{4}{7}$
\end{tabular}

After observing message $b$ any Receiver's belief that the ban is beneficial is $1 / 2$, so voting in favor of the proposal is consistent with sincere voting. Again, since all voters vote

\footnotetext{
${ }^{5}$ Rather than a single entity trying to persuade many agents, there are also models in which many entities try to persuade a single agent. Gentzkow and Kamenica (2017) show that the amount of information revealed increases with competition. Li and Norman (2018) consider a model both with simultaneous and sequential moves, where they show that it is possible to have a loss of information by the addition of a sender.

${ }^{6}$ Many single Receiver models which build on Kamenica and Gentzkow (2011) also consider a Sender or Receiver who has private information. Hedlund (2017) and Kosenko (2018) consider a Sender with private information, whereas in Kolotilin et al. (2017), there is a privately informed Receiver.

${ }^{7}$ We assume that the only source of information of a committee member is government. Tsakas and Tsakas (2017) consider a model where there exists noise due to exogenous information distortions and show that increasing noise cannot be beneficial to Sender.

${ }^{8}$ Alonso and Câmara (2016), Laclau and Renou (2016), and Alonso and Câmara (2018) all use public communication strategies.

${ }^{9}$ As each member's posterior is the same in this case, the situation is very similar to the situation in Kamenica and Gentzkow (2011).
} 
equally, this is an equilibrium. The probability that the proposal is being implemented is exactly the probability that signal $\mathbf{b}$ realizes. Hence, the acceptance probability of the proposal is $3 / 10 \cdot 1+7 / 10 \cdot 3 / 7=60 \%$.

Persuasion with private independent signals Instead of using a public signal, government could communicate privately with each committee member. ${ }^{10}$ Private communication can easily be achieved by any Sender using today's technology, especially via social media. So, suppose government employs a research strategy which is observed by the committee, but each member receives (conditional on the state) an independent private message. ${ }^{11}$ We can think of this situation as employing three biased researchers conducting three independent studies that are conditional on the true state. Each researcher reports the results of their study privately to one committee member, so that each committee member observes $b$ or $h$ with the following conditional probabilities.

\begin{tabular}{c|cc}
$\pi$ & $B$ & $H$ \\
\hline$b$ & 1 & $\frac{3}{7}$ \\
$h$ & 0 & $\frac{4}{7}$
\end{tabular}

The acceptance probability of the ban is $\frac{3}{10} \cdot 1+\frac{7}{10} \cdot\left(3 \cdot \frac{3}{7} \cdot \frac{3}{7} \cdot \frac{4}{7}+\frac{3}{7} \cdot \frac{3}{7} \cdot \frac{3}{7}\right) \approx 58 \%$, which is strictly less than in case of public communication.

Persuasion with private correlated signals Government's capabilities do not end with employing independent private signals; it can do better by using correlated private messages. ${ }^{12}$ Suppose that government hires two economists, one being honest and one partisan. The honest economist conducts a fully informative study and reports to one randomly selected committee member. The partisan conducts a biased study that will send $b$ if $B$ is the true state and send $b$ with probability $9 / 14$ if $H$ is the true state. He reports his results to the remaining two committee members. The following table captures the conditional probabilities for each of the five possible signal vectors being sent.

\footnotetext{
${ }^{10}$ Arieli and Babichenko (2019) also consider a multiple Receiver model with private communication for different utility functions of Sender.

${ }^{11}$ Wang (2013) considers private strategies with independent realizations and makes a comparison with public strategies.

${ }^{12}$ Bergemann and Morris (2016) consider correlation in an environment where players observe the information structure but also can observe additional private messages. Heese and Lauermann (2017) also consider correlated private messages where voters have heterogeneous and private preferences.
} 


\begin{tabular}{c|cc}
$\pi^{*}$ & $B$ & $H$ \\
\hline$(b, b, b)$ & 1 & 0 \\
$(b, b, h)$ & 0 & $\frac{3}{14}$ \\
$(b, h, b)$ & 0 & $\frac{3}{14}$ \\
$(h, b, b)$ & 0 & $\frac{3}{14}$ \\
$(h, h, h)$ & 0 & $\frac{5}{14}$
\end{tabular}

According to $\pi^{*}$ the probability of a Receiver to observe $b$, if the true state is $H$, is $3 / 7$. Hence, the posterior on the ban being beneficial after observing $b$ is $1 / 2$ as before. Thus, provided that voters act sincerely, government is able to increase the probability of implementing its preferred outcome to $3 / 10+7 / 10 \cdot 9 / 14=75 \%$ by sending private messages.

The swing voter's curse and the equilibrium constraint In the previous setting committee members know that the ban will be harmful to society upon receiving $h$, but are uncertain upon observing $b$. Still, given their posterior belief, each member votes for the proposal upon observing $b$ under sincere "Sender-preferred" voting. ${ }^{13}$ Yet, given communication strategy $\pi^{*}$, if all voters are sincere, each one becomes pivotal if and only if they have received $b$ and the ban is harmful. This phenomenon is an instance of the swing voter's curse (Feddersen and Pesendorfer, 1996). A swing voter is an agent whose vote is decisive, that is, a pivotal voter. The swing voter's curse refers to situations in which a partially informed voter is pivotal only when he should vote against his favorite outcome. ${ }^{14}$ Given communication strategy $\pi^{*}$ each committee member is better off voting against the ban after having observed $b$ : in this case either the state is $B$ and the other two members have observed $b$ as well (so he is not pivotal), or the state is $H$ and he is pivotal. In the former his vote has no effect on the outcome, and in the latter he is pivotal and should vote against the ban. Thus, sincere voting cannot be an equilibrium. It is, then, natural to ask: what can Sender achieve under the additional constraint that sincere voting be an equilibrium? We will characterize the optimal communication strategy under this constraint and analyze its effect on Sender's expected utility.

\subsection{Outline of the paper}

In Section 2 we introduce the necessary notation, define the voting game, and formulate Sender's problem. In Section 3 we focus on sincere voters. We first show that Sender can

\footnotetext{
${ }^{13}$ Sincere voting depends on first-order beliefs only. Mathevet et al. (forthcoming) consider higherorder beliefs and characterize the extent to which a Sender can manipulate agents' beliefs by disclosing information.

${ }^{14}$ There are empirical studies on the swing voter's curse such as Battaglini et al. (2010) and Grosser and Seebauer (2016). For a both theoretical and empirical approach on the swing voter's curse in the context of communication with experts, see Buechel and Mechtenberg (2019).
} 
restrict attention to straightforward communication strategies and then find the optimal communication strategy. In Section 4 we define the Bayes-Nash equilibrium in order to analyze the case of strategic voting. In Section 5, we find the optimal communication strategy under strategic voting. Finally, we conclude in Section 6.

\section{Preliminaries and Notation}

\subsection{Communication Strategies and Beliefs}

Let $N=\{1, \ldots, n\}$ be the set of Receivers and let $\Omega$ be a finite set of states of the world. Let $S_{i}$ be a finite set of messages Sender can send to Receiver $i \in N$ and let $S=\prod_{i \in N} S_{i}$ be the set of signals. A communication strategy is a function $\pi: \Omega \rightarrow \Delta(S)$ which maps each state of the world to a joint probability distribution over signal realizations. Denote the set of all communication strategies by $\Pi$. For each signal $s \in S$, let $s_{i} \in S_{i}$ denote the message for Receiver $i$. For each $s_{i}^{\prime} \in S_{i}$ and $\omega \in \Omega$, let

$$
\pi_{i}\left(s_{i}^{\prime} \mid \omega\right)=\sum_{s \in S: s_{i}=s_{i}^{\prime}} \pi(s \mid \omega),
$$

which is the probability that Receiver $i$ observes $s_{i}$ given that the true state is $\omega$. For any $\pi \in \Pi$ define $S^{\pi}=\{s \in S \mid \exists \omega \in \Omega: \pi(s \mid \omega)>0\}$. That is, $S^{\pi}$ consists of signals in $S$ which are sent with positive probability by $\pi$. Similarly, for each $\pi \in \Pi$ and $i \in N$, define $S_{i}^{\pi}=\left\{s_{i} \in S_{i} \mid \exists \omega \in \Omega: \pi_{i}\left(s_{i} \mid \omega\right)>0\right\}$ to be the set of messages Receiver $i$ observes with positive probability under $\pi$.

Throughout the paper we assume that Sender and Receivers share a common prior belief $\lambda^{0} \in \Delta^{\circ}(\Omega)$ about the true state of the world, where $\Delta^{\circ}(\Omega)$ denotes the set of strictly positive probability distributions on $\Omega$. Given $\lambda^{0} \in \Delta^{\circ}(\Omega)$ and $\pi \in \Pi$, a signal $s \in S^{\pi}$ generates the posterior belief vector $\lambda^{s} \in \Delta(\Omega)^{n}$ defined by

$$
\lambda_{i}^{s}(\omega)=\frac{\pi_{i}\left(s_{i} \mid \omega\right) \lambda^{0}(\omega)}{\sum_{\omega^{\prime} \in \Omega} \pi_{i}\left(s_{i} \mid \omega^{\prime}\right) \lambda^{0}\left(\omega^{\prime}\right)}, \quad i \in N, \omega \in \Omega .
$$

That is, $\lambda_{i}^{s}(\omega)$ denotes $i$ 's updated belief upon receiving $s_{i}$ that the true state is $\omega$. Let $\sigma \in \Delta\left(\Delta(\Omega)^{n}\right)$ be a distribution over belief vectors. A communication strategy $\pi \in \Pi$ induces $\sigma$ if for all $\lambda \in \operatorname{supp}(\sigma)$ it holds that

$$
\sigma(\lambda)=\sum_{s \in S^{\pi}: \lambda^{s}=\lambda} \sum_{\omega \in \Omega} \pi(s \mid \omega) \lambda^{0}(\omega)
$$

In words, $\sigma(\lambda)$ is the probability of posterior vector $\lambda .{ }^{15}$ We say that $\sigma$ is inducible if there exists a communication strategy which induces it. We call $\sigma$ finite if $|\operatorname{supp}(\sigma)|<\infty .{ }^{16}$

\footnotetext{
${ }^{15}$ Note that if $\lambda$ does not belong to $\operatorname{supp}(\sigma)$, then the equality in (1) remains valid as then the right-hand side is equal to 0 .

${ }^{16} \mathrm{As} S$ is finite, each inducible $\sigma$ is a finite distribution.
} 


\subsection{Voting Problems}

A voting problem is a tuple $\left(N, \Omega, Z,\left(A_{i}\right)_{i \in N}, k, v,\left(u_{i}\right)_{i \in N}, \lambda^{0}\right)$ of the set of Receivers $N$, the set of states $\Omega$, the set of outcomes $Z$, Receivers' action sets $A_{i}$ that determine the outcome together with integer quota $k \geq 1$, Sender's utility function $v: Z \times \Omega \rightarrow \mathbb{R}$, Receivers' utility functions $u_{i}: Z \times \Omega \rightarrow \mathbb{R}$ and a common prior $\lambda^{0}$.

From here we shall focus on a binary set of states, that is, $\Omega=\{X, Y\}$. We assume that there are two voting outcomes which we represent by $Z=\{x, y\}$ and that there are two possible actions for each Receiver. That is, for each $i \in N$ we assume $A_{i}=\{x, y\}$. Action $x$ means casting a vote in favor of $x$ and action $y$ a vote in favor of $y$.

Let $A=\prod_{i \in N} A_{i}$ be the space of action profiles. Let $z^{k}: A \rightarrow Z$ be a voting rule such that for each $a \in A$, we have

$$
z^{k}(a)= \begin{cases}x & \text { if }\left|i \in N: a_{i}=x\right| \geq k \\ y & \text { otherwise }\end{cases}
$$

So, $z^{k}(a)$ is the outcome of the vote when the action profile is $a$ and quota is $k \cdot{ }^{17}$ If $k=n$, then $z^{k}$ is the unanimity rule.

We assume that Sender's utility is independent of the state and only depends on the outcome. For any $z \in Z$, Sender's utility function is defined as

$$
v(z)= \begin{cases}1 & \text { if } z=x \\ 0 & \text { otherwise }\end{cases}
$$

That is, Sender wants to implement $x$ and does not care about the true state.

For each $i \in N$, let $u_{i}: Z \times \Omega \rightarrow\{0,1\}$ be the utility function of Receiver $i$, where $u_{i}(z, \omega)$ is the utility of $i$ when the implemented outcome is $z$ and the true state is $\omega$. Define $u_{i}$ by

$$
u_{i}(z, \omega)= \begin{cases}1 & \text { if }[z=x \text { and } \omega=X] \text { or }[z=y \text { and } \omega=Y] \\ 0 & \text { otherwise. }\end{cases}
$$

That is, Receivers want the implemented outcome to match the true state of the world.

\subsection{Persuasion}

Let a communication strategy $\pi \in \Pi$ be given. For each $i \in N$, let $\alpha_{i}^{\pi}: S_{i}^{\pi} \rightarrow A_{i}$ be the sincere action function of Receiver $i$. That is, $\alpha_{i}^{\pi}$ assigns the action that a sincere voter $i$ would choose upon observing $s_{i}$. More precisely, let

$$
\alpha_{i}^{\pi}\left(s_{i}\right)= \begin{cases}x & \text { if } \lambda_{i}^{s}(X) \geq \frac{1}{2} \\ y & \text { otherwise }\end{cases}
$$

\footnotetext{
${ }^{17}$ Note that any anonymous and monotonic voting rule is of this form.
} 
Note that $\alpha_{i}^{\pi}$ is the "Sender-preferred" sincere action function: Receivers vote in favor of Sender's preferred outcome in case they are indifferent. In particular, if $\lambda^{0}(X) \geq 1 / 2$, then interests of Sender and Receivers are aligned and there is no need for communication.

Let $\pi \in \Pi$ be a communication strategy and for any $i \in N$ let

$$
\begin{aligned}
& M_{i}^{x}(\pi)=\left\{m \in S_{i}^{\pi} \mid \alpha_{i}^{\pi}(m)=x\right\}, \\
& M_{i}^{y}(\pi)=\left\{m \in S_{i}^{\pi} \mid \alpha_{i}^{\pi}(m)=y\right\},
\end{aligned}
$$

which are the sets of messages in $S_{i}^{\pi}$ which make $i$ choose $x$ and $y$, respectively.

We say that Receiver $i$ is pivotal in $s \in S^{\pi}$ if for any $a_{i} \in A_{i}, z^{k}\left(a_{i}, \alpha_{-i}^{\pi}\left(s_{-i}\right)\right)=a_{i}$. That is, $i$ is pivotal in the voting game following $s$ if $i$ 's vote determines the voting outcome given that all $j \neq i$ vote sincerely. Define

$$
Z^{x}(\pi)=\left\{s \in S^{\pi} \mid z^{k}\left(\alpha^{\pi}(s)\right)=x\right\}
$$

as the set of signals in $S^{\pi}$ that lead to $x$ being implemented under sincere voting. Sender's expected utility given $\lambda^{0} \in \Delta^{\circ}(\Omega)$ and $\pi \in \Pi$ is the value of $\pi$ at $\lambda^{0}$ and denoted by $V^{\pi}\left(\lambda^{0}\right)$. The value $V^{\pi}\left(\lambda^{0}\right)$ equals the probability that $x$ is being implemented given $\lambda^{0}$ and $\pi$. That is,

$$
V^{\pi}\left(\lambda^{0}\right)=\mathbb{E}_{\lambda^{0}}\left[\mathbb{E}_{\pi}\left[v\left(z^{k}\left(\alpha^{\pi}(s)\right)\right)\right]\right]=\lambda^{0}(X) \sum_{s \in Z^{x}(\pi)} \pi(s \mid X)+\lambda^{0}(Y) \sum_{s \in Z^{x}(\pi)} \pi(s \mid Y) .
$$

Let $\Pi^{\prime} \subseteq \Pi$. We say that $\pi^{*} \in \Pi^{\prime}$ is optimal in $\Pi^{\prime}$ at $\lambda^{0} \in \Delta^{\circ}(\Omega)$ if

$$
V^{\pi^{*}}\left(\lambda^{0}\right)=\sup _{\pi \in \Pi^{\prime}} V^{\pi}\left(\lambda^{0}\right) .
$$

\section{Sincere Voting}

In our motivating example of a government wanting to ban cars on certain days of the year, the messages committee members observe are recommendations to either vote in favor of or against the ban, that is, for each $i \in N, S_{i}^{\pi} \subseteq A_{i}$. However, it could also be that messages are statistics about the pollution caused by excess car usage on which Receivers base their decision, rather than a recommended action, so that $S_{i}^{\pi}$ would not be a subset of $A_{i}$. In Section 4.1 we show that we can without loss of generality assume $S_{i}^{\pi} \subseteq A_{i}$ for all $i \in N$ when searching for the optimal communication strategy of Sender, a property known as straightforwardness of the communication strategy.

\subsection{Straightforward Communication Strategies}

Definition 3.1. A communication strategy $\pi \in \Pi$ is straightforward if for all $i \in N$ 
(i) $S_{i}^{\pi} \subseteq A_{i}$,

(ii) $\alpha_{i}^{\pi}\left(a_{i}\right)=a_{i}$ for all $a_{i} \in A_{i}$.

Denote the set of all straightforward communication strategies by $\Pi^{\mathrm{s}}$. Under a straightforward communication strategy, for each $i \in N$, the set of messages $i$ can observe with positive probability is a subset of his action set; thus, the communication strategy sends a "recommended" action to each Receiver. Condition (ii) requires that these recommendations be convincing in the sense that sincere voters follow their recommendations.

By definition, a communication strategy $\pi \in \Pi$ with $S^{\pi} \in\{x, y\}^{n}$ is straightforward if for any $i \in N$ and $s, t \in S^{\pi}$ with $s_{i}=x$ and $t_{i}=y$, we have $\lambda_{i}^{s}(X) \geq 1 / 2$ and $\lambda_{i}^{t}(Y)>1 / 2$. Hence, $\pi$ is straightforward if and only if for all $i \in N$

$$
\begin{aligned}
& \lambda^{0}(X) \pi_{i}(x \mid X) \geq \lambda^{0}(Y) \pi_{i}(x \mid Y), \\
& \lambda^{0}(X) \pi_{i}(y \mid X)<\lambda^{0}(Y) \pi_{i}(y \mid Y) .
\end{aligned}
$$

We show in Lemma 3.2 that most of the time we do not have to pay attention to (5). ${ }^{18}$

Lemma 3.2. Let $\lambda^{0}(X) \in(0,1 / 2)$. Then $\pi \in \Pi^{\mathrm{s}}$ if and only if $\pi$ satisfies (4).

Although focusing on straightforward communication strategies would simplify the problem, one important defect of doing so is the shrinkage in the set of inducible posteriors: it is not in general true that any inducible posterior can be induced by a straightforward communication strategy. We demonstrate this in the next example.

Example 3.3. Let $N=\{1,2\}$ and $\lambda^{0}(X)=1 / 3$. Let $S_{i}=\{w, x, y\}$ be the set of messages. Let the communication strategy $\hat{\pi} \in \Pi$ be given by

\begin{tabular}{c|cc}
$\hat{\pi}$ & $\omega=X$ & $\omega=Y$ \\
\hline$(w, x)$ & $\frac{1}{2}$ & 0 \\
$(w, y)$ & 0 & $\frac{1}{2}$ \\
$(w, w)$ & $\frac{1}{2}$ & $\frac{1}{2}$
\end{tabular}

A simple calculation of the posteriors shows that the support of the distribution $\hat{\sigma}$ induced by $\hat{\pi}$ is $\operatorname{supp}(\hat{\sigma})=\{(1 / 3,1),(1 / 3,0),(1 / 3,1 / 3)\}$, where a posterior belief vector $\lambda$ is represented by $\left(\lambda_{1}(X), \lambda_{2}(X)\right)$. The communication strategy $\hat{\pi}$ is not straightforward since Receiver 2 has more messages than actions, so that $S_{2}^{\pi} \nsubseteq A_{2}$. The reason we cannot find a straightforward communication strategy which induces $\hat{\sigma}$ is that Receiver 2 has three different posterior beliefs under $\hat{\sigma}$, whereas a straightforward communication strategy can induce at most $\left|A_{i}\right|=2$ different posterior beliefs.

\footnotetext{
${ }^{18}$ All proofs can be found in the appendix.
} 
In spite of this limitation of focusing on straightforward communication strategies, it is still without loss of generality to do so when solving the optimization problem in (3): there might not always exist a straightforward communication strategy which induces the same distribution as any given $\hat{\pi} \in \Pi$, but there always exists $\pi \in \Pi^{\mathrm{s}}$ which has at least the same value as $\hat{\pi}$.

Lemma 3.4. Let $\lambda^{0} \in \Delta^{\circ}(\Omega)$ and $\hat{\pi} \in \Pi$. Then there is $\pi \in \Pi^{\mathrm{s}}$ with $V^{\pi}\left(\lambda^{0}\right) \geq V^{\hat{\pi}}\left(\lambda^{0}\right)$.

If a certain value can be achieved, it can be achieved using a straightforward communication strategy. So, when searching for Sender's optimal communication strategy at any given $\lambda^{0}$, we can without loss of generality restrict our attention to straightforward communication strategies. We demonstrate Lemma 3.4 in the next example.

Example 3.5. Recall Example 3.3, where we showed that no straightforward communication strategy can induce $\hat{\sigma}$. Under $\hat{\pi}$, signals $(w, w)$ and $(w, y)$ lead to action profile $(y, y)$ and signal $(w, x)$ leads to action profile $(y, x)$. Assume that outcome $x$ is implemented if at least one of the agents votes for $x$, i.e., $k=1$. Then the probability that $x$ is implemented is

$$
V^{\hat{\pi}}\left(\lambda^{0}\right)=\lambda^{0}(X) \hat{\pi}((w, x) \mid X)+\lambda^{0}(Y) \hat{\pi}((w, x) \mid Y)=\frac{1}{3} \cdot \frac{1}{2}+\frac{2}{3} \cdot 0=\frac{1}{6} .
$$

Let $\pi \in \Pi$ be such that $S^{\pi}=\{(y, y),(y, x)\}$ and, for all $\omega \in \Omega$, let $\pi((y, y) \mid \omega)=$ $\hat{\pi}((w, w) \mid \omega)+\hat{\pi}((w, y) \mid \omega)$ and $\pi((y, x) \mid \omega)=\hat{\pi}((w, x) \mid \omega)$. Then

\begin{tabular}{c|cc}
$\pi$ & $\omega=X$ & $\omega=Y$ \\
\hline$(y, x)$ & $\frac{1}{2}$ & 0 \\
$(y, y)$ & $\frac{1}{2}$ & 1
\end{tabular}

We have $\operatorname{supp}(\sigma)=\{(1 / 3,1),(1 / 3,1 / 5)\} \neq \operatorname{supp}(\hat{\sigma})$. Yet, $x$ is implemented if Receivers have posterior $(1 / 3,1)$, that is if the signal realization is $(y, x)$. Thus, $x$ is implemented with probability

$$
V^{\pi}\left(\lambda^{0}\right)=\lambda^{0}(X) \pi((y, x) \mid X)+\lambda^{0}(Y) \pi((y, x) \mid Y)=\frac{1}{3} \cdot \frac{1}{2}+\frac{2}{3} \cdot 0=\frac{1}{6}
$$

as well.

\subsection{Optimal Public Communication}

We say that a communication strategy $\pi \in \Pi$ is public if for all $s \in S^{\pi}$ and all $i, j \in N$, $s_{i}=s_{j}$, that is, if all Receivers observe the same message under $\pi$. Since agents have homogeneous preferences, a public communication strategy either persuades all Receivers or none. Thus, the analysis of a multiple Receiver model with public communication 
strategies is very similar to the well-known analysis of the single Receiver model in Kamenica and Gentzkow (2011). Having that said, we proceed with a brief discussion of the former in order to allow for later references.

Let $\Pi^{\mathrm{p}}$ be the set of all public communication strategies. The most that Sender can achieve using public signals is

$$
V^{* \mathrm{p}}\left(\lambda^{0}\right)=\sup _{\pi \in \Pi^{\mathrm{p}}} V^{\pi}\left(\lambda^{0}\right)
$$

If the communication strategy $\hat{\pi}$ in Lemma 3.4 is public, then $\pi$ as constructed in the proof of the lemma is public as well. Hence, when searching for the optimal public communication strategy, it is no of loss of generality to consider only public straightforward communication strategies, that is, those $\pi \in \Pi$ with $S^{\pi} \subseteq\{\mathbf{x}, \mathbf{y}\} .{ }^{19}$

Proposition 3.6. Let $\lambda^{0} \in \Delta^{\circ}(\Omega)$. Let $\pi^{* \mathrm{p}}$ be given by

$$
\pi^{* \mathrm{p}}(s \mid \omega)= \begin{cases}1 & \text { if } s=\mathbf{x} \text { and } \omega=X \\ \min \left\{\frac{\lambda^{0}(X)}{\lambda^{0}(Y)}, 1\right\} & \text { if } s=\mathbf{x} \text { and } \omega=Y, \\ \max \left\{1-\frac{\lambda^{0}(X)}{\lambda^{0}(Y)}, 0\right\} & \text { if } s=\mathbf{y} \text { and } \omega=Y\end{cases}
$$

Then $\pi^{* \mathrm{p}}$ is optimal in $\Pi^{\mathrm{p}}$. In particular, $V^{* \mathrm{p}}\left(\lambda^{0}\right)=\min \left\{2 \lambda^{0}(X), 1\right\}$.

Via public communication Sender can achieve the value $2 \lambda^{0}(X)$. In the following sections we show how Sender can improve upon this by communicating privately with Receivers.

\subsection{Optimal Private Communication}

Our aim is to find a communication strategy which maximizes Sender's expected utility if Sender can use arbitrary private signals. In this case, the highest value she can achieve at any $\lambda^{0} \in \Delta^{\circ}(\Omega)$ is

$$
V^{*}\left(\lambda^{0}\right)=\sup _{\pi \in \Pi} V^{\pi}\left(\lambda^{0}\right)
$$

For the single Receiver case, Kamenica and Gentzkow (2011) show that it is optimal for Sender to send $x$ to the Receiver with probability 1 if the state is $X$. Lemma 3.7 shows that this result extends to the multiple Receiver case. ${ }^{20}$

\footnotetext{
${ }^{19}$ We denote by $\mathbf{x}, \mathbf{y} \in A$ the vectors $\left(x_{i}\right)_{i \in N}$ and $\left(y_{i}\right)_{i \in N}$, respectively.

${ }^{20}$ Our result also follows from Lemma 1 of Arieli and Babichenko (2019). In their model, agents want their action to match the true state, whereas in our model agents want the outcome of the vote to match the true state. However, as sincere voters operate under the assumption that their vote is decisive, the two optimization problems are equivalent. Nevertheless, for the sake of completeness, we provide a simple proof in the appendix.
} 
Lemma 3.7. Let $\lambda^{0} \in \Delta^{\circ}(\Omega)$ and $\hat{\pi} \in \Pi$. Then there is $\pi \in \Pi^{\mathrm{s}}$ such that for all $i \in N$ it holds that $\pi_{i}(x \mid X)=1$ and $V^{\pi}\left(\lambda^{0}\right) \geq V^{\hat{\pi}}\left(\lambda^{0}\right)$.

The voting rule $z^{k}$ is anonymous: only the total number of votes in favor of each outcome matters, not the identities of the voters who voted for each alternative. Hence, we can expect that communication strategies need not discriminate between Receivers either. In order to make this claim formal, let $B$ denote the set of all permutations on $N{ }^{21}$ For each $s \in S^{\pi}$ and each $b \in B$, denote by $s^{b}$ the unique signal vector with $s_{i}^{b}=s_{b(i)}$ for all $i \in N$. A communication strategy $\pi \in \Pi$ is called anonymous if $\pi(s \mid \omega)=\pi\left(s^{b} \mid \omega\right)$ for all bijections $b \in B$ and all $\omega \in \Omega$. The set of all straightforward and anonymous communication strategies is denoted by $\Pi^{\text {sa }}$. In the next lemma we show that Sender can indeed restrict her search for the optimal communication strategy to $\Pi^{\text {sa }}$.

Lemma 3.8. Let $\lambda^{0} \in \Delta^{\circ}(\Omega)$ and $\hat{\pi} \in \Pi$. Then there is $\pi \in \Pi^{\mathrm{sa}}$ with $V^{\pi}\left(\lambda^{0}\right) \geq V^{\hat{\pi}}\left(\lambda^{0}\right)$.

In the following, define for $\ell=0, \ldots, n$,

$$
S_{\ell}^{x}=\left\{s \in\{x, y\}^{n}:\left|\left\{j \in N: s_{j}=x\right\}\right|=\ell\right\} .
$$

That is, $S_{\ell}^{x}$ is the set of signals in which exactly $\ell$ Receivers observe $x$. An anonymous communication strategy $\pi$ can be represented by weights $q=\left(q_{0}, \ldots, q_{n}\right) \geq 0$ and $r=$ $\left(r_{0}, \ldots, r_{n}\right) \geq 0$ with $\sum_{\ell=0}^{n} q_{\ell}=\sum_{\ell=0}^{n} r_{\ell}=1$ such that

$$
\begin{aligned}
& \pi(s \mid X)=\left(\begin{array}{l}
n \\
\ell
\end{array}\right)^{-1} q_{\ell}, \quad s \in S_{\ell}^{x}, \\
& \pi(s \mid Y)=\left(\begin{array}{l}
n \\
\ell
\end{array}\right)^{-1} r_{\ell}, \quad s \in S_{\ell}^{x} .
\end{aligned}
$$

That is, $q_{\ell}$ is the probability that $x$ is sent to exactly $\ell$ Receivers if the state is $X$ and $r_{\ell}$ is the probability that $x$ is sent to exactly $\ell$ Receivers if the state is $Y$. Thus, the probabilities that an agent observes $x$ given that the state is $X$ and $Y$, respectively, are

$$
\begin{aligned}
& \pi_{i}(x \mid X)=\sum_{\ell=0}^{n} \sum_{s \in S_{\ell}^{x}: s_{i}=x}\left(\begin{array}{l}
n \\
\ell
\end{array}\right)^{-1} q_{\ell}=\sum_{\ell=0}^{n}\left(\begin{array}{l}
n-1 \\
\ell-1
\end{array}\right)\left(\begin{array}{l}
n \\
\ell
\end{array}\right)^{-1} q_{\ell}=\sum_{\ell=0}^{n} \frac{\ell}{n} q_{\ell} \\
& \pi_{i}(x \mid Y)=\sum_{\ell=0}^{n} \sum_{s \in S_{\ell}^{x}: s_{i}=x}\left(\begin{array}{l}
n \\
\ell
\end{array}\right)^{-1} r_{\ell}=\sum_{\ell=0}^{n}\left(\begin{array}{c}
n-1 \\
\ell-1
\end{array}\right)\left(\begin{array}{l}
n \\
\ell
\end{array}\right)^{-1} r_{\ell}=\sum_{\ell=0}^{n} \frac{\ell}{n} r_{\ell} .
\end{aligned}
$$

Recall that by Lemma 3.7, it is optimal to send $x$ to everyone with probability 1 if the state is $X$, that is, there is an optimal anonymous communication strategy with representation $(q, r)$ satisfying $q_{n}=1$. So, what remains is finding the optimal values for $r$. In the next proposition, we express $V^{*}\left(\lambda^{0}\right)$ as the solution to a simple linear optimization problem.

\footnotetext{
${ }^{21}$ A permutation on $N$ is a bijection $b: N \rightarrow N$.
} 
Proposition 3.9. Let $\lambda^{0} \in \Delta^{\circ}(\Omega)$. Then $V^{*}\left(\lambda^{0}\right)$ is the solution to

$$
\begin{aligned}
\max _{r_{0}, r_{k}} \lambda^{0}(X)+\lambda^{0}(Y) r_{k} & \\
\text { s.t. } r_{0} & \geq 0, \\
r_{k} & \geq 0, \\
r_{0}+r_{k} & =1, \\
\lambda^{0}(X)-\lambda^{0}(Y) \frac{k}{n} r_{k} & \geq 0 .
\end{aligned}
$$

The intuition behind Proposition 3.9 is as follows. Suppose the true state is $Y$ and message $x$ is sent to $\ell<k$ Receivers with positive probability. Under a straightforward communication strategy such a signal will not implement $x$ and can be replaced by $\mathbf{y}$. Suppose the true state is $Y$ and message $x$ is sent to $\ell>k$ Receivers, so $x$ is implemented under a straightforward communication strategy. Sending $x$ to exactly $k$ Receivers would have the same effect without violating the straightforwardness constraint. Hence, choosing $r$ such that $r_{0}+r_{k}=1$ is without loss of generality.

By Lemma 3.8 and Proposition 3.9, for any $\lambda^{0} \in \Delta^{\circ}(\Omega)$, it holds that

$$
V^{*}\left(\lambda^{0}\right)=\sup _{\pi \in \Pi} V^{\pi}\left(\lambda^{0}\right)=\max _{\pi \in \Pi^{\mathrm{sa}}} V^{\pi}\left(\lambda^{0}\right),
$$

i.e., there is an anonymous and straightforward communication strategy that achieves $V^{*}\left(\lambda^{0}\right)$. Such an optimal communication strategy is given in Theorem $3.10 .^{22}$

Theorem 3.10. Let $\pi^{*} \in \Pi^{\mathrm{sa}}$ with representation $\left(q^{*}, r^{*}\right)$ be given by

$$
\left(q_{n}^{*} ; r_{0}^{*}, r_{k}^{*}\right)= \begin{cases}(1 ; 0,1) & \text { if } \lambda^{0}(X) \geq \frac{k}{n+k}, \\ \left(1 ; 1-\frac{\lambda^{0}(X)}{\lambda^{0}(Y)} \frac{n}{k}, \frac{\lambda^{0}(X)}{\lambda^{0}(Y)} \frac{n}{k}\right) & \text { if } \lambda^{0}(X)<\frac{k}{n+k} .\end{cases}
$$

Then $\pi^{*}$ is optimal at $\lambda^{0}$. In particular, $V^{*}$ is given by

$$
V^{*}\left(\lambda^{0}\right)=\min \left\{\frac{n+k}{k} \lambda^{0}(X), 1\right\} .
$$

The communication strategy $\pi^{*}$ differs from $\pi^{* \mathrm{p}}$ only if the state is $Y$. By sending $x$ to $k$ rather than $n$ Receivers, Sender can increase the probability that at least $k$ voters observe $x$ without affecting each individual Receiver's probability of observing $x$, so the straightforwardness constraint remains satisfied.

Example 3.11. Let $n=3, k=2$, and $\lambda^{0}(X)=1 / 3$. Since $\lambda^{0}(X)=1 / 3<2 / 5=$ $k /(n+k)$, by Theorem 3.10, the optimal private communication strategy is given by $q_{3}^{*}=1, r_{0}^{*}=1 / 4$, and $r_{2}^{*}=3 / 4$. Alternatively, we can represent $\pi^{*}$ by

\footnotetext{
${ }^{22}$ Note that Theorem 3.10 is equivalent to Corollary 2 of Arieli and Babichenko (2019).
} 


\begin{tabular}{c|cc}
$\pi^{*}$ & $\omega=X$ & $\omega=Y$ \\
\hline$(x, x, x)$ & 1 & 0 \\
$(x, x, y)$ & 0 & $\frac{1}{4}$ \\
$(x, y, x)$ & 0 & $\frac{1}{4}$ \\
$(y, x, x)$ & 0 & $\frac{1}{4}$ \\
$(y, y, y)$ & 0 & $\frac{1}{4}$
\end{tabular}

Moreover, Sender is better off employing $\pi^{*}$ rather than $\pi^{* \mathrm{p}}$ as $V^{\pi^{*}}(1 / 3)=5 / 6>2 / 3=$ $V^{\pi^{* \mathrm{p}}}(1 / 3)$.

\section{$4 \quad$ Strategic Voting}

Recall the sincere voters from Example 3.11 and consider some fixed Receiver $i$. If the true state is $X$, all Receivers observe $x$ and, as they act sincerely, Receiver $i$ is not pivotal. If, on the other hand, the state is $Y$, he is pivotal upon observing $x$ as his vote will determine the outcome. Therefore, $i$ is better off choosing action $y$ upon observing $x$ : either the true state is indeed $X$ and his vote does not matter, or the state is $Y$ in which case voting for $y$ is strictly beneficial. This phenomenon has been coined the swing voter's curse: even though an agent believes that the true state is $X$, his rational action is to vote in favor of $y$. The following example illustrates this point by calculating Receiver's expected utility from voting $x$ after having observed $x$.

Example 4.1. Recall the communication strategy $\pi^{*}$ in Example 3.11. The expected utility of Receiver $i$ from voting for $x$ conditional on having observed message $x$ and provided that all other Receivers vote sincerely is given by

$$
\begin{aligned}
\lambda_{i}^{\mathbf{x}}(X) & \sum_{s \in S^{\pi}: s_{i}=x} \frac{\pi\left(\left(x, s_{-i}\right) \mid X\right)}{\pi_{i}(x \mid X)} u_{i}(x, X)+\lambda_{i}^{\mathbf{x}}(Y) \sum_{s \in S^{\pi}: s_{i}=x} \frac{\pi\left(\left(x, s_{-i}\right) \mid Y\right)}{\pi_{i}(x \mid Y)} u_{i}(x, Y) \\
= & \frac{1}{2} \cdot 1 \cdot 1+0=\frac{1}{2} .
\end{aligned}
$$

On the other hand, his expected utility from voting for $y$ conditional on having observing message $x$ and provided that all other Receivers vote sincerely is given by

$$
\begin{aligned}
\lambda_{i}^{\mathbf{x}}(X) & \sum_{s \in S^{\pi}: s_{i}=x} \frac{\pi\left(\left(x, s_{-i}\right) \mid X\right)}{\pi_{i}(x \mid X)} u_{i}(x, X)+\lambda_{i}^{\mathbf{x}}(Y) \sum_{s \in S^{\pi}: s_{i}=x} \frac{\pi\left(\left(x, s_{-i}\right) \mid Y\right)}{\pi_{i}(x \mid Y)} u_{i}(y, Y) \\
= & \frac{1}{2} \cdot 1 \cdot 1+\frac{1}{2} \cdot\left(\frac{1 / 4}{1 / 2} \cdot 1+\frac{1 / 4}{1 / 2} \cdot 1\right)=1 .
\end{aligned}
$$

Hence, choosing action $y$ upon observing message $x$ is optimal for Receiver $i$. 
Requiring that sincere voting indeed be in the voters' best interest poses a new constraint on Sender's optimization problem. This constraint is formalized in the following definition.

Definition 4.2. For any $\pi \in \Pi$, we say that $\alpha^{\pi}=\left(\alpha_{i}^{\pi}\right)_{i \in N}$ constitutes a Bayes-Nash equilibrium if for all $i \in N, s^{\prime} \in S^{\pi}$, and $a_{i} \in A_{i}$, it holds that

$$
\begin{aligned}
\sum_{\omega \in \Omega} \lambda_{i}^{s^{\prime}}(\omega) & \sum_{s \in S^{\pi}: s_{i}=s_{i}^{\prime}} \frac{\pi\left(\left(s_{i}^{\prime}, s_{-i}\right) \mid \omega\right)}{\pi_{i}\left(s_{i}^{\prime} \mid \omega\right)} u_{i}\left(z^{k}\left(\alpha_{i}^{\pi}\left(s_{i}^{\prime}\right), \alpha_{-i}^{\pi}\left(s_{-i}\right)\right), \omega\right) \\
\geq & \sum_{\omega \in \Omega} \lambda_{i}^{s^{\prime}}(\omega) \sum_{s \in S^{\pi}: s_{i}=s_{i}^{\prime}} \frac{\pi\left(\left(s_{i}^{\prime}, s_{-i}\right) \mid \omega\right)}{\pi_{i}\left(s_{i}^{\prime} \mid \omega\right)} u_{i}\left(z^{k}\left(a_{i}, \alpha_{-i}^{\pi}\left(s_{-i}\right)\right), \omega\right) .
\end{aligned}
$$

Given a signal realization and other Receivers' sincere actions, (13) requires that a Receiver not be able to profitably deviate from $\alpha_{i}^{\pi}\left(s_{i}\right)$. One can easily observe that if a signal $s$ is sent under which no agents are pivotal, there is no difference in the expected utility from voting for $x$ and $y$ for any Receiver. Thus, an investigation of the BNE constraint (13) targets signals in which some agent is pivotal. We therefore define, for each $i \in N$, $\pi \in \Pi$, and $m \in S_{i}^{\pi}$ the set

$$
P^{i, m}(\pi)=\left\{s \in S^{\pi}: s_{i}=m \text { and } i \text { is pivotal }\right\} .
$$

We start by simplifying the BNE constraint (13) by means of these sets.

Lemma 4.3. Let $\lambda^{0} \in \Delta^{\circ}(\Omega)$ and $\pi \in \Pi$. Then $\alpha^{\pi}=\left(\alpha_{i}^{\pi}\right)_{i \in N}$ is a BNE if and only if for all $i \in N, m \in M_{i}^{x}(\pi)$, and $m^{\prime} \in M_{i}^{y}(\pi)$ it holds that

$$
\begin{aligned}
& \lambda^{0}(X) \sum_{s \in P^{i, m}(\pi)} \pi(s \mid X) \geq \lambda^{0}(Y) \sum_{s \in P^{i, m}(\pi)} \pi(s \mid Y), \\
& \lambda^{0}(Y) \sum_{s \in P^{i, m^{\prime}}(\pi)} \pi(s \mid Y) \geq \lambda^{0}(X) \sum_{s \in P^{i, m^{\prime}}(\pi)} \pi(s \mid X) .
\end{aligned}
$$

Because of the swing voter's curse, sincere voting cannot be an equilibrium under the optimal private communication strategy $\pi^{*}$ if $k \leq n-1$ : upon observing $x$, Receivers are pivotal with probability 1 if the state is $Y$ and not pivotal if the state is $X$. Hence, voting for $y$ is optimal. However, sincere voting is a BNE under $\pi^{*}$ if $z^{k}$ is the unanimity voting rule, i.e., if $k=n$.

Proposition 4.4. Let $\lambda^{0} \in \Delta^{\circ}(\Omega)$ and let $\pi^{*} \in \Pi$ be the optimal private communication strategy at $\lambda^{0}$ in Theorem 3.10. Then $\alpha^{\pi^{*}}$ is a BNE if and only if $k=n$.

If $k=n$, then $\pi^{*}$ is a public communication strategy. But even if $k \leq n-1$, any public communication strategy implements sincere voting as BNE: in this case no agent is ever pivotal and the two constraints in Lemma 4.3 are trivially satisfied. 


\begin{tabular}{c|cc}
$\pi^{*}$ & $\omega=X$ & $\omega=Y$ \\
\hline$(x, x, x, x, x)$ & 1 & 0 \\
$(x, x, x, y, y)$ & 0 & $\frac{1}{12}$ \\
$(x, x, y, y, x)$ & 0 & $\frac{1}{12}$ \\
$(x, y, y, x, x)$ & 0 & $\frac{1}{12}$ \\
$(y, y, x, x, x)$ & 0 & $\frac{1}{12}$ \\
$(y, x, y, x, x)$ & 0 & $\frac{1}{12}$ \\
$(y, x, x, y, x)$ & 0 & $\frac{1}{12}$ \\
$(y, x, x, x, y)$ & 0 & $\frac{1}{12}$ \\
$(x, y, x, y, x)$ & 0 & $\frac{1}{12}$ \\
$(x, x, y, x, y)$ & 0 & $\frac{1}{12}$ \\
$(x, y, x, x, y)$ & 0 & $\frac{1}{12}$ \\
$(y, y, y, y, y)$ & 0 & $\frac{1}{6}$
\end{tabular}

Table 1: Communication strategy $\pi^{*}$.

\begin{tabular}{c|cc}
$\pi$ & $\omega=X$ & $\omega=Y$ \\
\hline$(x, x, x, x, x)$ & 0 & 0 \\
$(x, x, x, y, y)$ & $\frac{1}{10}$ & $\frac{1}{20}$ \\
$(x, x, y, y, x)$ & $\frac{1}{10}$ & $\frac{1}{20}$ \\
$(x, y, y, x, x)$ & $\frac{1}{10}$ & $\frac{1}{20}$ \\
$(y, y, x, x, x)$ & $\frac{1}{10}$ & $\frac{1}{20}$ \\
$(y, x, y, x, x)$ & $\frac{1}{10}$ & $\frac{1}{20}$ \\
$(y, x, x, y, x)$ & $\frac{1}{10}$ & $\frac{1}{20}$ \\
$(y, x, x, x, y)$ & $\frac{1}{10}$ & $\frac{1}{20}$ \\
$(x, y, x, y, x)$ & $\frac{1}{10}$ & $\frac{1}{20}$ \\
$(x, x, y, x, y)$ & $\frac{1}{10}$ & $\frac{1}{20}$ \\
$(x, y, x, x, y)$ & $\frac{1}{10}$ & $\frac{1}{20}$ \\
$(y, y, y, y, y)$ & 0 & $\frac{1}{2}$
\end{tabular}

Table 2: Communication strategy $\pi$.

Suppose $\pi^{*}$ from Theorem 3.10 is being implemented and Receiver $i$ observes $x$. Then it is in his best interest to vote $y$ as we have seen in Example 4.1. Given the constraints in Lemma 4.3, there are two intuitive ways to adjust $\pi^{*}$ which ensure sincere voting be an equilibrium: following the observation $x$ one either needs that $i$ 's probability of being pivotal is higher if the state is $X$ (at $\pi^{*}$ this probability is 0 ) or lower if the state is $Y$ (at $\pi^{*}$ this probability is 1 ). We illustrate both approaches in the following example.

Example 4.5. Let $n=5, k=3$, and $\lambda^{0}(X)=1 / 3$. The optimal private communication strategy $\pi^{*}$ is given in Table 1 and a communication strategy $\pi$ with higher probability of being pivotal if the state is $X$ is given in Table 2 . Note that $\pi$ is chosen such that $r_{3}$ is as high as possible, subject to the constraints imposed by straightforwardness (4) and BNE constraints (14) and (15). For any $i \in N$, we have $\lambda_{i}^{\mathbf{x}}(X)=1 / 2$, so that $\alpha_{i}^{\pi}(x)=x$. A simple calculation shows that upon receiving $x$ Receivers are indifferent between following their recommendation and deviating. That is, (14) holds with equality. Moreover, agents are never pivotal upon observing $y$, so (15) is trivially satisfied. Therefore, $\alpha^{\pi}$ is a BNE. However, $\pi$ does not improve upon $\pi^{* p}$ :

$$
V^{\pi}\left(\frac{1}{3}\right)=\frac{1}{3} \sum_{s \in Z^{x}(\pi)} \pi(s \mid X)+\frac{2}{3} \sum_{s \in Z^{x}(\pi)} \pi(s \mid Y)=\frac{1}{3} \cdot 1+\frac{2}{3} \cdot \frac{1}{2}=\frac{2}{3}=V^{* \mathrm{p}}\left(\frac{1}{3}\right) .
$$

Now consider communication strategy $\pi^{\prime}$ in Table 3 where $x$ is sent to $k+1=4$ agents rather than a minimal winning coalition consisting of $k=3$ agents if the state is $Y$. Clearly, $P^{i, x}(\pi)=P^{i, y}(\pi)=\emptyset$, that is, an agent's probability of being pivotal is always 0. Thus, (14) and (15) hold with equality, so that $\alpha^{\pi^{\prime}}$ is a BNE. For all $i \in N$, we have 


\begin{tabular}{c|cc}
$\pi^{\prime}$ & $\omega=X$ & $\omega=Y$ \\
\hline$(x, x, x, x, x)$ & 1 & 0 \\
$(x, x, x, x, y)$ & 0 & $\frac{1}{8}$ \\
$(x, x, x, y, x)$ & 0 & $\frac{1}{8}$ \\
$(x, x, y, x, y)$ & 0 & $\frac{1}{8}$ \\
$(x, y, x, x, y)$ & 0 & $\frac{1}{8}$ \\
$(y, x, x, x, x)$ & 0 & $\frac{1}{8}$ \\
$(y, y, y, y, y)$ & 0 & $\frac{3}{8}$
\end{tabular}

Table 3: Communication strategy $\pi^{\prime}$.

$\lambda_{i}^{\mathbf{x}}(X)=1 / 2$ and $\lambda_{i}^{\mathbf{y}}(X)=0$. So, $\pi^{\prime}$ is straightforward as well. Moreover,

$$
V^{\pi^{\prime}}\left(\frac{1}{3}\right)=\frac{1}{3} \sum_{s \in Z^{x}\left(\pi^{\prime}\right)} \pi^{\prime}(s \mid X)+\frac{2}{3} \sum_{s \in Z^{x}\left(\pi^{\prime}\right)} \pi^{\prime}(s \mid Y)=\frac{1}{3} \cdot 1+\frac{2}{3} \cdot \frac{5}{8}=\frac{3}{4}>\frac{2}{3}=V^{* \mathrm{p}}\left(\frac{1}{3}\right) .
$$

that is, $\pi^{\prime}$ improves upon $\pi^{* \mathrm{p}}$.

\section{Optimal Communication under Strategic Voting}

In Proposition 4.4 we have characterized an optimal communication strategy that implements sincere voting as BNE for the case $k=n$. In this section we will, therefore, focus on $k \leq n-1$. Let $\Pi^{\mathrm{e}}$ be the set of all communication strategies under which sincere voting constitutes a BNE, i.e., $\Pi^{\mathrm{e}}=\left\{\pi \in \Pi \mid \alpha^{\pi}\right.$ is a BNE $\}$. Sender's problem is to find $\pi \in \Pi^{\mathrm{e}}$ which maximizes her expected utility. That is,

$$
V^{* \mathrm{e}}\left(\lambda^{0}\right)=\sup _{\pi \in \Pi^{\mathrm{e}}} V^{\pi}\left(\lambda^{0}\right) .
$$

We first show that even under the additional BNE constraints (14) and (15) we can still restrict attention to straightforward and anonymous communication strategies. Let $\Pi^{\text {sae }}$ denote the set of communication strategies in $\Pi^{\mathrm{e}}$ that are straightforward and anonymous.

Lemma 5.1. Let $\lambda^{0} \in \Delta^{\circ}(\Omega)$ and $\hat{\pi} \in \Pi^{\mathrm{e}}$. Then there is $\pi \in \Pi^{\text {sae }}$ with $V^{\pi}\left(\lambda^{0}\right) \geq V^{\hat{\pi}}\left(\lambda^{0}\right)$.

In Proposition 5.4 we will express $V^{* e}\left(\lambda^{0}\right)$ as the solution to a linear optimization problem. Lemma 5.1 allows us again to represent the constraints in terms of parameters $q$ and $r$. In particular, the two BNE constraints in Lemma 4.3 are equivalent to

$$
\begin{aligned}
\lambda^{0}(X) q_{k} & \geq \lambda^{0}(Y) r_{k}, \\
\lambda^{0}(Y) r_{k-1} & \geq \lambda^{0}(X) q_{k-1} .
\end{aligned}
$$

A trivial way to satisfy these constraints is to choose $q_{k-1}=q_{k}=r_{k}=0$. The next lemma shows that this can be done without loss of generality. 
Lemma 5.2. Let $\lambda^{0} \in \Delta^{\circ}(\Omega), \hat{\pi} \in \Pi^{\text {sae }}$, and $k \leq n-1$. Then there exists $\pi \in \Pi^{\text {sae }}$ with representation $(q, r)$ such that $q_{k-1}=q_{k}=r_{k}=0$ and $V^{\pi}\left(\lambda^{0}\right) \geq V^{\hat{\pi}}\left(\lambda^{0}\right)$.

Lemma 5.2 is in stark contrast to Theorem 3.10: when no BNE constraint is present, it is in Sender's best interest to make Receivers pivotal upon observing $x$ if the state is $Y$, i.e., $r_{k}>0 .^{23}$ We show next how the problem can be further simplified.

Lemma 5.3. Let $\lambda^{0} \in \Delta^{\circ}(\Omega), \hat{\pi}=(\hat{q}, \hat{r}) \in \Pi^{\text {sae }}$, and $k \leq n-1$. Then there exists $\pi \in \Pi^{\text {sae }}$ with representation $(q, r)$ such that $q_{n}=1, r_{\ell}=0$ for all $\ell \neq 0, k+1$, and $V^{\pi}\left(\lambda^{0}\right) \geq V^{\hat{\pi}}\left(\lambda^{0}\right)$.

When choosing $q$ and $r$ as suggested in Lemma 5.3, constraints (16) and (17) are clearly satisfied. Thus, the optimization problem that Sender faces under the additional BNE constraint is very similar to the one in Proposition 3.9.

Proposition 5.4. Let $\lambda^{0} \in \Delta^{\circ}(\Omega)$ and $k \leq n-1$. Then $V^{* e}\left(\lambda^{0}\right)$ is the solution to

$$
\begin{aligned}
\max _{r_{0}, r_{k+1}} \lambda^{0}(X)+\lambda^{0}(Y) r_{k+1} & \\
\text { s.t. } r_{0} & \geq 0, \\
r_{k+1} & \geq 0, \\
r_{0}+r_{k+1} & =1, \\
\lambda^{0}(X)-\lambda^{0}(Y) \frac{k+1}{n} r_{k+1} & \geq 0 .
\end{aligned}
$$

Interestingly, Proposition 5.4 differs from Proposition 3.9 only in that the BNE constraint shifts weight from $r_{k}$ to $r_{k+1}$. As all constraints are weak inequalities, and because of Lemma 5.1, for any $\lambda^{0} \in \Delta^{\circ}(\Omega)$, it holds that

$$
V^{* \mathrm{e}}\left(\lambda^{0}\right)=\max _{\pi \in \Pi^{\mathrm{see}}} V^{\pi}\left(\lambda^{0}\right),
$$

so there is $\pi \in \Pi^{\text {sae }}$ that achieves the optimal value. Such an optimal communication strategy is given in Theorem 5.5.

Theorem 5.5. Let $\lambda^{0} \in \Delta^{\circ}(\Omega)$. If $k \leq n-1$, then the communication strategy $\pi^{* \mathrm{e}} \in \Pi^{\text {sae }}$ with representation $\left(q^{* \mathrm{e}}, r^{* \mathrm{e}}\right)$ given by

$$
\left(q_{n}^{* \mathrm{e}} ; r_{0}^{* \mathrm{e}}, r_{k+1}^{* \mathrm{e}}\right)= \begin{cases}(1 ; 0,1) & \text { if } \lambda^{0}(X) \geq \frac{k+1}{n+k+1} \\ \left(1 ; 1-\frac{\lambda^{0}(X)}{\lambda^{0}(Y)} \frac{n}{k+1}, \frac{\lambda^{0}(X)}{\lambda^{0}(Y)} \frac{n}{k+1}\right) & \text { if } \lambda^{0}(X)<\frac{k+1}{n+k+1}\end{cases}
$$

is optimal at $\lambda^{0}$ in $\Pi^{\mathrm{e}}$. In particular, the optimal value function is given by

$$
V^{* e}\left(\lambda^{0}\right)=\min \left\{\frac{n+k+1}{k+1} \lambda^{0}(X), 1\right\} .
$$

\footnotetext{
${ }^{23}$ We should mention that Lemma 5.2 is not true for $k=n$, as in that case it is optimal for Sender to make all Receivers observe $x$ if the state is $X$, i.e., $q_{k}=q_{n}=1$.
} 
Theorem 5.5 covers the case that voting is not unanimous. Lemma 5.3 established that for an optimal communication strategy $\pi^{* \mathrm{e}}$ it holds that $q_{n}^{* \mathrm{e}}=1$. If $\lambda^{0}(X) \geq(k+1) /(n+k+1)$, then it is optimal to send $x$ with probability 1 to a coalition of $k+1$ Receivers if the state is $Y$. So, Receivers are not pivotal in either state and $x$ is implemented with probability 1 . If $\lambda^{0}(X)<(k+1) /(n+k+1)$, then $r_{0}^{* e}>0$. As there is a positive probability that $x$ is not implemented, the value of the optimal communication strategy is strictly less than 1 .

If $k \geq n-1$, Proposition 4.4 and Theorem 5.5 together imply that a public signal is optimal. Yet, if $k=n-1$, there is another, very different, optimal communication strategy: in Example 4.5 we presented $\pi$ which had the property that agents were pivotal upon observing $x$ in either state, and we showed that this strategy obtains the same value as the optimal public communication strategy $\pi^{* \mathrm{p}}$. The fact that $\pi^{* \mathrm{p}}$ is optimal under the BNE constraint if $k=n-1$ motivates the following corollary.

Corollary 5.6. Let $k=n-1$ and $\lambda^{0} \in \Delta^{\circ}(\Omega)$. The communication strategy represented by $q_{k}=1, r_{k}=\lambda^{0}(X) / \lambda^{0}(Y) q_{k}$, and $r_{0}=1-r_{k}$ is optimal.

In order to prove the corollary, one only needs to verify that the constraints are satisfied and that this communication strategy performs as well as $\pi^{* \mathrm{p}}$. The corollary highlights a fundamentally different way to achieve the optimal value in this setting.

If $k \leq n-1$, designing the communication strategy so that sincere behavior constitutes a BNE is not without a cost for Sender, namely losing some probability of implementing her preferred outcome.

Corollary 5.7. Let $\lambda^{0} \in \Delta^{\circ}(\Omega)$.

(i) If $k \leq n-1$ then $V^{* \mathrm{p}}\left(\lambda^{0}\right) \leq V^{* \mathrm{e}}\left(\lambda^{0}\right) \leq V^{*}\left(\lambda^{0}\right) \leq \frac{k+1}{k} \frac{n+k}{n+k+1} V^{* \mathrm{e}}\left(\lambda^{0}\right)$.

(ii) If $k=n$ then $V^{*}\left(\lambda^{0}\right)=V^{* \mathrm{p}}\left(\lambda^{0}\right)=V^{* \mathrm{e}}\left(\lambda^{0}\right)$.

In Figure 1, we plot the value functions $V^{*}, V^{* \mathrm{e}}$ and $V^{* \mathrm{p}}$ for $n=5$ and $k=3$. Sender's expected utility is highest when she does not care about implementing sincere voting as a $\mathrm{BNE}$, which is given by the straight line in Figure 1. Under the BNE constraint she cannot prevent a decrease in the probability of implementing her preferred outcome and ends up with a lower expected utility, i.e., $V^{*}\left(\lambda^{0}\right)>V^{* \mathrm{e}}\left(\lambda^{0}\right)$ for $\lambda^{0}(X)<(k+1) /(n+k+1)$.

It is easy to see that the value of an optimal communication strategy which implements sincere voting as BNE is decreasing in the quota.

Corollary 5.8. Let $\lambda^{0} \in \Delta^{\circ}(\Omega)$. The optimal value $V^{* \mathrm{e}}\left(\lambda^{0}\right)$ is weakly decreasing in $k$.

This result is intuitive, since persuasion becomes more difficult for Sender as the number of Receivers that have to be convinced increases. In particular, the value of the optimal communication strategy which implements sincere voting as BNE decreases with $k$ and equals the value of the optimal public communication strategy if $k \geq n-1$. In contrast, if we fix the quota, then Sender can implement $x$ with probability 1 when the number of 


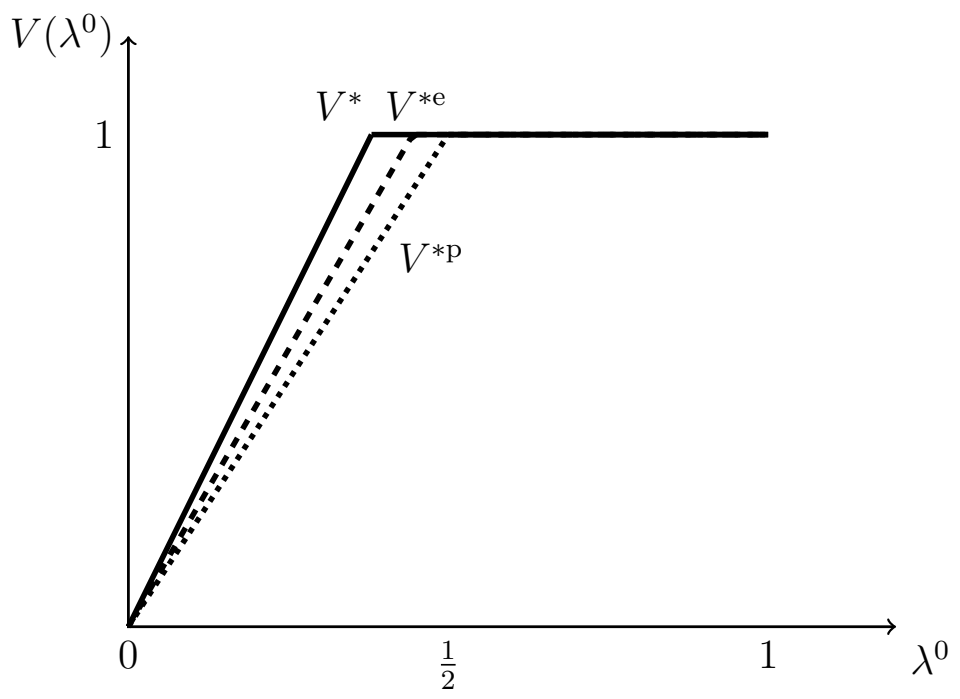

Figure 1: Values of $\pi^{*}, \pi^{* \mathrm{e}}$ and $\pi^{* \mathrm{p}}$ for $n=5$ and $k=3$.

Receivers tends to infinity, i.e., $\lim _{n \rightarrow \infty} V^{* e}\left(\lambda^{0}\right)=1$. Finally, if the the quota is a fixed ratio of the number of voters as in case of majority voting, Theorem 5.5 reveals that the cost of implementing sincere voting as BNE decreases as the population increases. In particular, if $k=\lceil p n\rceil$ for some $p \in(0,1]$, one has, for all $\lambda^{0} \in \Delta^{\circ}(\Omega)$,

$$
\lim _{n \rightarrow \infty} V^{* \mathrm{e}}\left(\lambda^{0}\right)=\lim _{n \rightarrow \infty} V^{*}\left(\lambda^{0}\right)= \begin{cases}1 & \text { if } \lambda^{0}(X) \geq \frac{p}{1+p} \\ \frac{1+p}{p} \lambda^{0}(X) & \text { if } \lambda^{0}(X)<\frac{p}{1+p}\end{cases}
$$

\section{Conclusion}

This paper investigates Bayesian persuasion where prior to a vote, a Sender attempts to persuade some homogeneous Receivers to vote for her favorite outcome. Motivated by social media that allow for targeted communication with individual receivers, we allow Sender to use private messages that may be correlated. While it is indeed beneficial for Sender to employ a private communication strategy with correlated messages rather than a public communication strategy, this might lead to situations in which Receivers should not vote according to their belief, that is, situations in which sincere voting is not an equilibrium. We show that the most efficient way for Sender to ensure that sincere voting be in the best interest of each Receiver is to send signals after which nobody is pivotal. That is, instead of persuading minimal winning coalitions, Sender should target slightly larger coalitions. This comes at a cost in terms of success probability, but this cost decreases as the population increases. 


\section{A Proofs}

Proof of Lemma 3.2. It is sufficient to show that (4) implies (5). So, let (4) be satisfied. Then

$$
\begin{aligned}
\lambda^{0}(X) \pi_{i}(y \mid X) & =\lambda^{0}(X)\left(1-\pi_{i}(x \mid X)\right) \\
& \stackrel{(4)}{\leq} \lambda^{0}(X)-\lambda^{0}(Y) \pi_{i}(x \mid Y) \\
& =\lambda^{0}(X)-\lambda^{0}(Y)+\lambda^{0}(Y) \pi_{i}(y \mid Y) \\
& <\lambda^{0}(Y) \pi_{i}(y \mid Y),
\end{aligned}
$$

where the first inequality follows from (4) and the last inequality holds since $\lambda^{0}(X)<$ $1 / 2<\lambda^{0}(Y)$. So, $\pi$ satisfies (5). Hence, $\pi$ is straightforward.

Proof of Lemma 3.4. If $\lambda^{0}(X) \geq 1 / 2$, let $\pi \in \Pi^{\mathrm{s}}$ be defined as $\pi(x \mid \omega)=1$ for all $\omega \in$ $\Omega$ and observe that $V^{\pi}\left(\lambda^{0}\right)=1 \geq V^{\hat{\pi}}\left(\lambda^{0}\right)$. Let $\lambda^{0}(X) \in(0,1 / 2)$. For each action profile $a \in A$, define $S^{a}(\hat{\pi})=\left\{s \in S^{\hat{\pi}} \mid \alpha^{\hat{\pi}}(s)=a\right\}$, which is the set of signals in $S^{\hat{\pi}}$ that lead to action profile $a$ under sincere voting. Note that for $a \neq b$, we have $S^{a}(\hat{\pi}) \cap S^{b}(\hat{\pi})=\emptyset$. Define a communication strategy $\pi \in \Pi$ by $\pi(a \mid \omega)=\sum_{s \in S^{a}(\hat{\pi})} \hat{\pi}(s \mid \omega)$ for each $\omega \in \Omega$ and $a \in A$, and note that $S_{i}^{\pi} \subseteq A_{i}$ for all $i \in N$. Fix $i \in N$. Then, for any $\omega \in \Omega$ and $a_{i} \in S_{i}^{\pi}$,

$$
\pi_{i}\left(a_{i} \mid \omega\right)=\sum_{t \in S^{\pi}: t_{i}=a_{i}} \pi(t \mid \omega)=\sum_{t \in S^{\pi}: t_{i}=a_{i}} \sum_{s \in S^{t}(\hat{\pi})} \hat{\pi}(s \mid \omega) .
$$

Recall that for each $i \in N$ we have $M_{i}^{x}(\hat{\pi})=\left\{m \in S_{i}^{\hat{\pi}} \mid \alpha_{i}^{\hat{\pi}}(m)=x\right\}$, which is the set of messages after which $i$ chooses action $x$ under sincere voting. Since $\lambda^{0}(X) \in(0,1 / 2)$, by Lemma 3.2 it is sufficient to show that $\pi$ satisfies (4).

Claim: It holds that

$$
\bigcup_{t \in S^{\pi}: t_{i}=x} S^{t}(\hat{\pi})=\bigcup_{m \in M_{i}^{x}(\hat{\pi})}\left\{t^{\prime} \in S^{\hat{\pi}}: t_{i}^{\prime}=m\right\}
$$

and both unions are over disjoint sets.

Proof. Let $s \in \bigcup_{t \in S^{\pi}: t_{i}=x} S^{t}(\hat{\pi})$. Then, there exists $t \in S^{\pi}$ with $t_{i}=x$ such that $s \in S^{t}(\hat{\pi})$. Thus, by the definition of $S^{t}(\pi), \alpha_{i}^{\hat{\pi}}\left(s_{i}\right)=x$, so that $s_{i} \in M_{i}^{x}(\hat{\pi})$. In particular, $s \in\left\{t^{\prime} \in S^{\hat{\pi}}: t_{i}^{\prime}=s_{i}\right\} \subseteq \bigcup_{m \in M_{i}^{x}(\hat{\pi})}\left\{t^{\prime} \in S^{\hat{\pi}}: t_{i}^{\prime}=m\right\}$. For the converse, suppose $s \in$ $\bigcup_{m \in M_{i}^{x}(\hat{\pi})}\left\{t^{\prime} \in S^{\hat{\pi}}: t_{i}^{\prime}=m\right\}$. Then there exists $m \in M_{i}^{x}(\hat{\pi})$ with $s_{i}=m$. Let $t=$ $\alpha^{\hat{\pi}}(s) \in A$ and observe that $t_{i}=\alpha_{i}^{\hat{\pi}}(m)=x$. Since by construction $s \in S^{t}(\hat{\pi})$ and $t \in S^{\pi}$, it holds that $s \in \bigcup_{t \in S^{\pi}: t_{i}=x} S^{t}(\hat{\pi})$.

As noted before, for any $a, b \in S^{\pi}$ with $a \neq b$ we have $S^{a}(\hat{\pi}) \cap S^{b}(\hat{\pi})=\emptyset$. Moreover, for any $m, m^{\prime} \in M_{i}^{x}(\hat{\pi})$ with $m \neq m^{\prime}$, we have $\left\{t^{\prime} \in S^{\hat{\pi}}: t_{i}^{\prime}=m\right\} \cap\left\{t^{\prime} \in S^{\hat{\pi}}: t_{i}^{\prime}=m^{\prime}\right\}=\emptyset$. Thus, both unions are over disjoint sets. This proves the claim. 
Let $M_{i}^{x}(\hat{\pi})=\left\{m_{1}, \ldots, m_{L}\right\}$ for some $L \geq 1$. For each $\ell \in\{1, \ldots, L\}, m_{\ell} \in M_{i}^{x}(\hat{\pi})$ and $s \in S^{\hat{\pi}}$ with $s_{i}=m_{\ell}$, let

$$
\begin{aligned}
c_{\ell} & =\sum_{t \in S^{\hat{\pi}}: t_{i}=m_{\ell}} \hat{\pi}(t \mid X) \lambda^{0}(X), \\
d_{\ell} & =\sum_{t \in S^{\hat{\pi}}: t_{i}=m_{\ell}} \hat{\pi}(t \mid X) \lambda^{0}(X)+\sum_{t \in S^{\hat{\pi}}: t_{i}=m_{\ell}} \hat{\pi}(t \mid Y) \lambda^{0}(Y),
\end{aligned}
$$

and note that $c_{\ell} / d_{\ell}=\lambda_{i}^{s}(X) \geq 1 / 2$ since $\alpha_{i}^{\hat{\pi}}\left(m_{\ell}\right)=x$. Thus, it holds that $d_{\ell} \leq 2 c_{\ell}$. Let $s^{\prime} \in S^{\pi}$ be such that $s_{i}^{\prime}=x$. We have that

$$
\begin{aligned}
\lambda_{i}^{t}(X) & =\frac{\pi_{i}(x \mid X) \lambda^{0}(X)}{\pi_{i}(x \mid X) \lambda^{0}(X)+\pi_{i}(x \mid Y) \lambda^{0}(Y)} \\
& =\frac{\sum_{t \in S^{\pi}: t_{i}=x} \sum_{s \in S^{t}(\hat{\pi})} \hat{\pi}(s \mid X) \lambda^{0}(X)}{\sum_{t \in S^{\pi}: t_{i}=x} \sum_{s \in S^{t}(\hat{\pi})} \hat{\pi}(s \mid X) \lambda^{0}(X)+\sum_{t \in S^{\pi}: t_{i}=x} \sum_{s \in S^{t}(\hat{\pi})} \hat{\pi}(s \mid Y) \lambda^{0}(Y)} \\
& \stackrel{(22)}{=} \frac{\sum_{m \in M_{i}^{x}(\hat{\pi})} \sum_{t \in S^{\hat{\pi}}: t_{i}=m} \hat{\pi}(t \mid X) \lambda^{0}(X)}{\sum_{m \in M_{i}^{x}(\hat{\pi})} \sum_{t \in S^{\hat{\pi}}: t_{i}=m} \hat{\pi}(t \mid X) \lambda^{0}(X)+\sum_{m \in M_{i}^{x}(\hat{\pi})} \sum_{t \in S^{\hat{\pi}}: t_{i}=m} \hat{\pi}(t \mid Y) \lambda^{0}(Y)} \\
& =\frac{c_{1}+\cdots+c_{L}}{d_{1}+\cdots+d_{L}} \geq \frac{c_{1}+\cdots+c_{L}}{2\left(c_{1}+\cdots+c_{L}\right)}=\frac{1}{2} .
\end{aligned}
$$

Thus, for any $m \in M_{i}^{x}(\hat{\pi})$, we have $\alpha_{i}^{\pi}(x)=\alpha_{i}^{\hat{\pi}}(m)=x$. Hence, $\pi$ satisfies $(4)$.

What remains to be shown is that $\pi$ has at least the same value as $\hat{\pi}$. By definition of $Z^{x}(\pi)$ it holds that

$$
\sum_{a \in Z^{x}(\pi)} \pi(a \mid \omega)=\sum_{a \in Z^{x}(\pi)} \sum_{s \in S^{a}(\hat{\pi})} \hat{\pi}(s \mid \omega)=\sum_{s \in Z^{x}(\hat{\pi})} \hat{\pi}(s \mid \omega) .
$$

Thus, we have

$$
\begin{aligned}
V^{\pi}\left(\lambda^{0}\right) & =\lambda^{0}(X) \sum_{s \in Z^{x}(\pi)} \pi(s \mid X)+\lambda^{0}(Y) \sum_{s \in Z^{x}(\pi)} \pi(s \mid Y) \\
& =\lambda^{0}(X) \sum_{s \in Z^{x}(\hat{\pi})} \hat{\pi}(s \mid X)+\lambda^{0}(Y) \sum_{s \in Z^{x}(\hat{\pi})} \hat{\pi}(s \mid Y) \\
& =V^{\hat{\pi}}\left(\lambda^{0}\right)
\end{aligned}
$$

Proof of Proposition 3.6. If $\lambda^{0}(X) \geq 1 / 2$, then $V^{\pi^{* \mathrm{p}}}\left(\lambda^{0}\right)=1$. Let $\lambda^{0}(X)<1 / 2$. When searching for an optimal $\pi \in \Pi^{\mathrm{ps}}$, we can also assume without loss of generality that $\pi(\mathbf{x} \mid X)=1$, as $V^{\pi}$ is increasing in $\pi(\mathbf{x} \mid X)$ and setting $\pi(\mathbf{x} \mid X)=1$ makes it easier to satisfy the straightforwardness constraint. It holds now that $\pi$ is straightforward if and 
only if $\pi(\mathbf{x} \mid Y) \leq \lambda^{0}(X) / \lambda^{0}(Y)$. Since the value is increasing in $\pi(\mathbf{x} \mid Y)$, it is optimal for Sender to choose $\pi(\mathbf{x} \mid Y)=\lambda^{0}(X) / \lambda^{0}(Y)$. The optimal value for any $\lambda^{0} \in \Delta^{\circ}(\Omega)$ is then given by

$$
V^{* \mathrm{p}}\left(\lambda^{0}\right)=\lambda^{0}(X) \pi^{* \mathrm{p}}(\mathbf{x} \mid X)+\lambda^{0}(Y) \pi^{* \mathrm{p}}(\mathbf{x} \mid Y)=\lambda^{0}(X)+\lambda^{0}(Y) \frac{\lambda^{0}(X)}{\lambda^{0}(Y)}=2 \lambda^{0}(X) .
$$

Proof of Lemma 3.7. If $\lambda^{0}(X) \geq 1 / 2$, then let $\pi \in \Pi^{\mathrm{s}}$ be defined as $\pi(x \mid \omega)=1$ for all $\omega \in \Omega$ and observe that $V^{\pi}\left(\lambda^{0}\right)=1 \geq V^{\hat{\pi}}\left(\lambda^{0}\right)$. Let $\lambda^{0}(X)<1 / 2$. By Lemma 3.4, we can assume without loss of generality that $\hat{\pi} \in \Pi^{\mathrm{s}}$. Thus, by Lemma $3.2, \lambda^{0}(X) \hat{\pi}_{i}(x \mid X) \geq$ $\lambda^{0}(Y) \hat{\pi}_{i}(x \mid Y)$ for all $i \in N$. Let $\pi \in \Pi^{\mathrm{s}}$ be the communication strategy such that for all $i \in N$ and $s_{i} \in S_{i}, \pi_{i}\left(s_{i} \mid Y\right)=\hat{\pi}_{i}\left(s_{i} \mid Y\right)$ and $\pi_{i}(x \mid X)=1$. That is, signal probabilities in state $Y$ remain the same, but $\pi$ sends $x$ to every Receiver with probability 1 if the state is $X$. Then

$$
\lambda^{0}(X) \pi_{i}(x \mid X) \geq \lambda^{0}(X) \hat{\pi}_{i}(x \mid X) \geq \lambda^{0}(Y) \hat{\pi}_{i}(x \mid Y)=\lambda^{0}(Y) \pi_{i}(x \mid Y),
$$

where the second inequality holds since $\hat{\pi} \in \Pi^{\mathrm{s}}$. By Lemma 3.2, $\pi$ is straightforward. Finally,

$$
\begin{aligned}
V^{\pi}\left(\lambda^{0}\right) & =\lambda^{0}(X)+\lambda^{0}(Y) \sum_{s \in Z^{x}(\pi)} \pi(s \mid Y) \geq \lambda^{0}(X) \sum_{s \in Z^{x}(\hat{\pi})} \hat{\pi}(s \mid X)+\lambda^{0}(Y) \sum_{s \in Z^{x}(\hat{\pi})} \hat{\pi}(s \mid Y) \\
& =V^{\hat{\pi}}\left(\lambda^{0}\right),
\end{aligned}
$$

which completes the proof.

Proof of Lemma 3.8. If $\lambda^{0}(X) \geq 1 / 2$, then let $\pi \in \Pi^{\text {sa }}$ be defined as $\pi(x \mid \omega)=1$ for all $\omega \in \Omega$ and observe that $V^{\pi}\left(\lambda^{0}\right)=1 \geq V^{\hat{\pi}}\left(\lambda^{0}\right)$. Let $\lambda^{0}(X)<1 / 2$. By Lemma 3.4, assume without loss of generality that $\hat{\pi}$ is straightforward. For each $\omega \in \Omega, s \in S$, and $b \in B$ define $\pi^{b}$ by $\pi^{b}(s \mid \omega)=\hat{\pi}\left(s^{b} \mid \omega\right)$, so that $S^{\pi^{b}}=\left\{s^{b^{-1}} \mid s \in S^{\hat{\pi}}\right\}$. Let $\pi \in \Pi$ be defined by

$$
\pi(s \mid \omega)=\frac{1}{n !} \sum_{b \in B} \pi^{b}(s \mid \omega), \quad \omega \in \Omega, s \in S .
$$

Clearly, $\pi$ is anonymous. We next show that $\pi$ is straightforward. Fix $i \in N$. Then

$$
\begin{aligned}
\pi_{i}(x \mid X) & =\sum_{s \in S^{\pi}: s_{i}=x} \pi(s \mid X)=\sum_{s \in S^{\pi}: s_{i}=x} \frac{1}{n !} \sum_{b \in B} \pi^{b}(s \mid X) \\
& =\frac{1}{n !} \sum_{b \in B} \sum_{s \in S^{\pi}: s_{i}=x} \hat{\pi}\left(s^{b} \mid X\right)=\frac{1}{n !} \sum_{b \in B} \sum_{s \in S^{\pi}: s_{b(i)}=x} \hat{\pi}(s \mid X) \\
& =\frac{1}{n !} \sum_{b \in B} \hat{\pi}_{b(i)}(x \mid X) .
\end{aligned}
$$


By Lemma 3.2, one has $\lambda^{0}(X) \hat{\pi}_{b(i)}(x \mid X) \geq \lambda^{0}(Y) \hat{\pi}_{b(i)}(x \mid Y)$ for all $b \in B$. Thus,

$$
\lambda^{0}(X) \pi_{i}(x \mid X)=\lambda^{0}(X) \frac{1}{n !} \sum_{b \in B} \hat{\pi}_{b(i)}(x \mid X) \geq \lambda^{0}(Y) \frac{1}{n !} \sum_{b \in B} \hat{\pi}_{b(i)}(x \mid Y)=\lambda^{0}(Y) \pi_{i}(x \mid Y) .
$$

Hence, $\pi$ satisfies (4) and, by Lemma 3.2, is straightforward. Finally,

$$
\begin{aligned}
V^{\pi}\left(\lambda^{0}\right) & =\lambda^{0}(X) \sum_{s \in Z^{x}(\pi)} \pi(s \mid X)+\lambda^{0}(Y) \sum_{s \in Z^{x}(\pi)} \pi(s \mid Y) \\
& =\lambda^{0}(X) \sum_{s \in Z^{x}(\pi)} \frac{1}{n !} \sum_{b \in B} \pi^{b}(s \mid X)+\lambda^{0}(Y) \sum_{s \in Z^{x}(\pi)} \frac{1}{n !} \sum_{b \in B} \pi^{b}(s \mid Y) \\
& =\lambda^{0}(X) \frac{1}{n !} \sum_{b \in B} \sum_{s \in Z^{x}\left(\pi^{b}\right)} \pi^{b}(s \mid X)+\lambda^{0}(Y) \frac{1}{n !} \sum_{b \in B} \sum_{s \in Z^{x}\left(\pi^{b}\right)} \pi^{b}(s \mid Y) \\
& =\lambda^{0}(X) \frac{1}{n !} \sum_{b \in B} \sum_{s \in Z^{x}(\hat{\pi})} \hat{\pi}(s \mid X)+\lambda^{0}(Y) \frac{1}{n !} \sum_{b \in B} \sum_{s \in Z^{x}(\hat{\pi})} \hat{\pi}(s \mid Y) \\
& =\lambda^{0}(X) \sum_{s \in Z^{x}(\hat{\pi})} \hat{\pi}(s \mid X)+\lambda^{0}(Y) \sum_{s \in Z^{x}(\hat{\pi})} \hat{\pi}(s \mid Y) \\
& =V^{\hat{\pi}}\left(\lambda^{0}\right) .
\end{aligned}
$$

where the fourth equation holds because $Z^{x}\left(\pi^{b}\right)=\left\{s^{b^{-1}} \mid s \in Z^{x}(\hat{\pi})\right\}$.

Proof of Proposition 3.9. If $\lambda^{0}(X) \geq 1 / 2$, then the solution to the linear optimization problem in Proposition 3.9 is given by $\left(r_{0}^{*}, r_{k}^{*}\right)=(0,1)$, leading to $V^{*}\left(\lambda^{0}\right)=1$ as desired. Let $\lambda^{0}(X)<1 / 2$. First note that the communication strategy $\pi$ as constructed in the proof of Lemma 3.7 satisfies anonymity if $\hat{\pi}$ is anonymous. Hence, by Lemma 3.7, $V^{*}\left(\lambda^{0}\right)$ can be obtained by an anonymous and straightforward communication strategy with $q_{n}=1$. We first show that it is no loss of generality to have $r_{\ell}=0$ for all $\ell \neq 0, k$. For this purpose let $\hat{\pi} \in \Pi^{\text {sa }}$ be a communication strategy with representation $(\hat{q}, \hat{r})$. Let $\pi \in \Pi^{\text {sa }}$ with representation $(q, r)$ be defined as $q_{n}=1$, $r_{0}=\hat{r}_{0}+\cdots+\hat{r}_{k-1}$ and $r_{k}=\hat{r}_{k}+\cdots+\hat{r}_{n}$. Then $\sum_{s \in Z^{x}(\pi)} \pi(s \mid X)=\sum_{s \in Z^{x}(\hat{\pi})} \hat{\pi}(s \mid X)$ and $\sum_{s \in Z^{x}(\pi)} \pi(s \mid Y)=\sum_{s \in Z^{x}(\hat{\pi})} \hat{\pi}(s \mid Y)$, so that $V^{\pi}\left(\lambda^{0}\right)=V^{\hat{\pi}}\left(\lambda^{0}\right)$. Therefore, Sender can restrict attention to weights $r_{0}$ and $r_{k}$ that satisfy the Constraints (9)-(11). By Lemma $3.2, \pi$ is straightforward if and only if

$$
0 \leq \lambda^{0}(X) \pi_{i}(x \mid X)-\lambda^{0}(Y) \pi_{i}(x \mid Y)=\lambda^{0}(X)-\lambda^{0}(Y) \frac{k}{n} r_{k},
$$

which is equivalent to (12). The objective function is, thus,

$$
\begin{aligned}
V^{\pi}\left(\lambda^{0}\right) & =\lambda^{0}(X) \sum_{s \in Z^{x}(\pi)} \pi(s \mid X)+\lambda^{0}(Y) \sum_{s \in Z^{x}(\pi)} \pi(s \mid Y)=\lambda^{0}(X) \cdot 1+\lambda^{0}(Y) r_{k} \\
& =\lambda^{0}(X)+\lambda^{0}(Y) r_{k} .
\end{aligned}
$$


Proof of Theorem 3.10. Sender's maximization problem is given in Proposition 3.9. As the objective function is increasing in $r_{k}$, Constraints (11) and (12) imply

$$
r_{k}^{*}=\min \left\{\frac{\lambda^{0}(X)}{\lambda^{0}(Y)} \frac{n}{k}, 1\right\}= \begin{cases}1 & \text { if } \lambda^{0}(X) \geq \frac{k}{n+k} \\ \frac{\lambda^{0}(X)}{\lambda^{0}(Y)} \frac{n}{k} & \text { if } \lambda^{0}(X)<\frac{k}{n+k}\end{cases}
$$

Thus, the optimal value for any $\lambda^{0} \in \Delta^{\circ}(\Omega)$ is

$$
V^{\pi^{*}}\left(\lambda^{0}\right)=\lambda^{0}(X)+\lambda^{0}(Y) r_{k}= \begin{cases}1 & \text { if } \lambda^{0}(X) \geq \frac{k}{n+k} \\ \frac{n+k}{k} \lambda^{0}(X) & \text { if } \lambda^{0}(X)<\frac{k}{n+k}\end{cases}
$$

Proof of Lemma 4.3. Let $i \in N$ be fixed. For any $s \in S^{\pi}$ it holds that

$$
\begin{aligned}
& u_{i}\left(z^{k}\left(x, \alpha_{-i}^{\pi}\left(s_{-i}\right)\right), X\right)-u_{i}\left(z^{k}\left(y, \alpha_{-i}^{\pi}\left(s_{-i}\right)\right), X\right)= \begin{cases}1 & \text { if } i \text { is pivotal at } s, \\
0 & \text { if } i \text { is not pivotal at } s,\end{cases} \\
& u_{i}\left(z^{k}\left(y, \alpha_{-i}^{\pi}\left(t_{-i}\right)\right), Y\right)-u_{i}\left(z^{k}\left(x, \alpha_{-i}^{\pi}\left(t_{-i}\right)\right), Y\right)= \begin{cases}1 & \text { if } i \text { is pivotal at } s, \\
0 & \text { if } i \text { is not pivotal at } s .\end{cases}
\end{aligned}
$$

For given $m \in M_{i}^{x}(\pi)$, let $s^{\prime} \in S^{\pi}$ be such that $s_{i}^{\prime}=m$ and let $c=\sum_{\omega \in \Omega} \lambda^{0}(\omega) \pi_{i}\left(s_{i}^{\prime} \mid \omega\right)$. Then

$$
\begin{aligned}
& \lambda^{0}(X) \sum_{s \in P^{i, m}(\pi)} \pi(s \mid X)-\lambda^{0}(Y) \sum_{s \in P^{i, m}(\pi)} \pi(s \mid Y) \\
& =c\left(\frac{\lambda^{0}(X) \pi_{i}\left(s_{i}^{\prime} \mid X\right)}{c} \sum_{s \in P^{i, m}(\pi)} \frac{\pi(s \mid X)}{\pi_{i}\left(s_{i}^{\prime} \mid X\right)}-\frac{\lambda^{0}(Y) \pi_{i}\left(s_{i}^{\prime} \mid Y\right)}{c} \sum_{s \in P^{i, m}(\pi)} \frac{\pi(s \mid Y)}{\pi_{i}\left(s_{i}^{\prime} \mid Y\right)}\right) \\
& =c\left(\lambda_{i}^{s^{\prime}}(X) \sum_{s \in P^{i, m}(\pi)} \frac{\pi(s \mid X)}{\pi_{i}\left(s_{i}^{\prime} \mid X\right)}\left(u_{i}\left(z^{k}\left(x, \alpha_{-i}^{\pi}\left(s_{-i}\right)\right), X\right)-u_{i}\left(z^{k}\left(y, \alpha_{-i}^{\pi}\left(s_{-i}\right)\right), X\right)\right)\right. \\
& \left.-\lambda_{i}^{s^{\prime}}(Y) \sum_{s \in P^{i, m}(\pi)} \frac{\pi(s \mid Y)}{\pi_{i}\left(s_{i}^{\prime} \mid Y\right)}\left(u_{i}\left(z^{k}\left(y, \alpha_{-i}^{\pi}\left(s_{-i}\right)\right), Y\right)\right)-u_{i}\left(z^{k}\left(x, \alpha_{-i}^{\pi}\left(s_{-i}\right)\right), Y\right)\right) \\
& =c \sum_{\omega \in \Omega} \lambda_{i}^{s^{\prime}}(\omega) \sum_{s \in S^{\pi}: s_{i}=s_{i}^{\prime}} \frac{\pi(s \mid \omega)}{\pi_{i}\left(s_{i}^{\prime} \mid \omega\right)}\left(u_{i}\left(z^{k}\left(x, \alpha_{-i}^{\pi}\left(s_{-i}\right)\right), \omega\right)-u_{i}\left(z^{k}\left(y, \alpha_{-i}^{\pi}\left(s_{-i}\right)\right), \omega\right)\right)
\end{aligned}
$$


and one shows similarly that for given $m^{\prime} \in M_{i}^{x}(\pi)$ and $t^{\prime} \in S^{\pi}$ with $t_{i}^{\prime}=m^{\prime}$

$$
\begin{aligned}
& \lambda^{0}(Y) \sum_{t \in P^{i, m^{\prime}}(\pi)} \pi(t \mid Y)-\lambda^{0}(X) \sum_{t \in P^{i, m^{\prime}}(\pi)} \pi(s \mid X) \\
= & c^{\prime} \sum_{\omega \in \Omega} \lambda_{i}^{t^{\prime}}(\omega) \sum_{t \in S^{\pi}: t_{i}=t_{i}^{\prime}} \frac{\pi(t \mid \omega)}{\pi_{i}\left(t_{i}^{\prime} \mid \omega\right)}\left(u_{i}\left(z^{k}\left(y, \alpha_{-i}^{\pi}\left(t_{-i}\right)\right), \omega\right)-u_{i}\left(z^{k}\left(x, \alpha_{-i}^{\pi}\left(t_{-i}\right)\right), \omega\right)\right),
\end{aligned}
$$

where $c^{\prime}=\sum_{\omega \in \Omega} \lambda^{0}(\omega) \pi_{i}\left(t_{i}^{\prime} \mid \omega\right)$. Thus, (13) holds if and only if (14) and (15) hold.

Proof of Proposition 4.4. Let $i \in N$ and suppose first that $k \leq n-1$. Then upon receiving $x, i$ is pivotal if the state is $Y$ but not pivotal if the state is $X$. Thus, we have $\sum_{s \in P^{i, x}\left(\pi^{*}\right)} \pi^{*}(s \mid X)=0$ and $\sum_{s \in P^{i, x}\left(\pi^{*}\right)} \pi^{*}(s \mid Y)>0$. If $\alpha^{\pi^{*}}$ were a BNE at $\pi^{*},(14)$ would imply that: $0 \geq \lambda^{0}(Y) \sum_{s \in P^{i, x}\left(\pi^{*}\right)} \pi^{*}(s \mid Y)>0$, which is impossible.

Let $k=n$. Since $i$ is not pivotal upon observing $y$, (15) is trivially satisfied, and we only need to check (14). By Theorem 3.10 we have $q_{n}^{*}=1$ and $r_{n}^{*}=\min \left\{\lambda^{0}(X) / \lambda^{0}(Y), 1\right\}$. Thus,

$$
\lambda^{0}(X) \sum_{s \in P^{i, x}\left(\pi^{*}\right)} \pi^{*}(s \mid X)=\lambda^{0}(X)=\lambda^{0}(Y) \frac{\lambda^{0}(X)}{\lambda^{0}(Y)} \geq \lambda^{0}(Y) r_{n}^{*}=\lambda^{0}(Y) \sum_{s \in P^{i, x}\left(\pi^{*}\right)} \pi^{*}(s \mid Y) .
$$

Hence, (14) is satisfied and $\alpha^{\pi^{*}}$ is a BNE.

Proof of Lemma 5.1. If $\lambda^{0}(X) \geq 1 / 2$, then consider $\pi \in \Pi^{\text {sae }}$ with representation $(q, r)$ such that $q_{n}=1$ and $r_{k+1}=1$. Observe that $V^{\pi}\left(\lambda^{0}\right)=1 \geq V^{\hat{\pi}}\left(\lambda^{0}\right)$. Let $\lambda^{0}(X)<$ $1 / 2$. We first show that without loss of generality we can assume $\hat{\pi}$ to be anonymous. Also, without loss of generality, we assume that for all $i, j \in N$ with $i \neq j$, we have $S_{i}^{\hat{\pi}} \cap S_{j}^{\hat{\pi}}=\emptyset$, i.e., different Receivers observe different messages. Let $\pi^{\prime} \in \Pi$ be defined as

$$
\pi^{\prime}(s \mid \omega)=\frac{1}{n !} \sum_{b \in B} \hat{\pi}\left(s^{b} \mid \omega\right), \quad \omega \in \Omega, s \in S .
$$

We showed in the proof of Lemma 3.8 that $\pi^{\prime} \in \Pi^{\mathrm{a}}$ and has value greater than or equal to $\hat{\pi}$. It is therefore sufficient to show that $\alpha^{\pi^{\prime}}$ is a BNE. Let $i \in N$ and $m \in M_{i}^{x}\left(\pi^{\prime}\right)$. Let $j \in N$ be the unique Receiver such that $m \in S_{j}^{\hat{\pi}}$. Then

$$
\sum_{s \in P^{i, m}\left(\pi^{\prime}\right)} \sum_{b \in B} \hat{\pi}\left(s^{b} \mid X\right)=\sum_{s \in P^{i, m}\left(\pi^{\prime}\right)} \sum_{b \in B: b(i)=j} \hat{\pi}\left(s^{b} \mid X\right)=(n-1) ! \sum_{s \in P^{j, m}(\hat{\pi})} \hat{\pi}(s \mid X) .
$$

Therefore,

$$
\begin{aligned}
\lambda^{0}(X) \sum_{s \in P^{i, m}\left(\pi^{\prime}\right)} \pi^{\prime}(s \mid X) & =\lambda^{0}(X) \frac{1}{n !} \sum_{s \in P^{i, m}\left(\pi^{\prime}\right)} \sum_{b \in B} \hat{\pi}\left(s^{b} \mid X\right) \stackrel{(24)}{=} \lambda^{0}(X) \frac{1}{n} \sum_{s \in P^{j, m}(\hat{\pi})} \hat{\pi}(s \mid X) \\
& \geq \lambda^{0}(Y) \frac{1}{n} \sum_{s \in P^{j, m}(\hat{\pi})} \hat{\pi}(s \mid Y)=\lambda^{0}(Y) \sum_{s \in P^{i, m}\left(\pi^{\prime}\right)} \pi^{\prime}(s \mid Y),
\end{aligned}
$$


where the inequality comes from Lemma 4.3 and the fact that $\alpha^{\hat{\pi}}$ is a BNE. A similar argument holds for $m^{\prime} \in M_{i}^{y}\left(\pi^{\prime}\right)$. It follows that $\alpha^{\pi^{\prime}}$ is a BNE. Thus, we can assume without loss of generality that $\hat{\pi}$ is anonymous.

Recall that for each action profile $a \in A, S^{a}(\hat{\pi})=\left\{s \in S^{\hat{\pi}} \mid \alpha^{\hat{\pi}}(s)=a\right\}$. For each $a \in A$ and $\omega \in \Omega$, define $\pi(a \mid \omega)=\sum_{s \in S^{a}(\hat{\pi})} \hat{\pi}(s \mid \omega)$. We showed in the proof of Lemma 3.4 that $\pi$ is straightforward and $V^{\pi}\left(\lambda^{0}\right)=V^{\hat{\pi}}\left(\lambda^{0}\right)$. So, what remains to show is that $\alpha^{\pi}$ is a BNE.

Claim: For all $i \in N$ it holds that

$$
\bigcup_{a \in P^{i, x}(\pi)} S^{a}(\hat{\pi})=\bigcup_{m \in M_{i}^{x}(\hat{\pi})} P^{i, m}(\hat{\pi}) .
$$

and the unions on both sides of the equation are over disjoint sets.

Proof. Let $s \in \bigcup_{a \in P^{i, x}(\pi)} S^{a}(\hat{\pi})$. Then, there exists $a \in S^{\pi}$ with $a_{i}=x$ such that $\alpha^{\hat{\pi}}(s)=a$ and $i$ is pivotal in $s$. Since $a_{i}=x$, we have $\alpha_{i}^{\hat{\pi}}\left(s_{i}\right)=a_{i}=x$, hence $s_{i} \in M_{i}^{x}(\hat{\pi})$, so $s \in \bigcup_{m \in M_{i}^{x}(\hat{\pi})} P^{i, m}(\hat{\pi})$. For the converse, let $s \in \bigcup_{m \in M_{i}^{x}(\hat{\pi})} P^{i, m}(\hat{\pi})$. Then, $s_{i} \in M_{i}^{x}(\hat{\pi})$ and $i$ is pivotal in $s$. Let $a=\alpha^{\hat{\pi}}(s)$, so that $s \in S^{a}(\hat{\pi})$. Since $s_{i} \in M_{i}^{x}(\hat{\pi})$, we have $a_{i}=\alpha_{i}^{\hat{\pi}}\left(s_{i}\right)=x$, so that $a \in P^{i, x}(\hat{\pi})$, hence $s \in \bigcup_{a \in P^{i, x}(\pi)} S^{a}(\hat{\pi})$. For the same reasons as in the proof of Lemma 3.8 both unions are over disjoint sets.

Since the unions on both sides of (25) are disjoint, we have

$$
\begin{aligned}
\lambda^{0}(X) \sum_{a \in P^{i, x}(\pi)} \pi(a \mid X) & =\lambda^{0}(X) \sum_{a \in P^{i, x}(\pi)} \sum_{t \in S^{a}(\hat{\pi})} \hat{\pi}(t \mid X) \stackrel{(25)}{=} \lambda^{0}(X) \sum_{m \in M_{i}^{x}(\hat{\pi})} \sum_{s \in P^{i, m}(\hat{\pi})} \hat{\pi}(s \mid X) \\
& \geq \lambda^{0}(Y) \sum_{m \in M_{i}^{x}(\hat{\pi})} \sum_{s \in P^{i, m}(\hat{\pi})} \hat{\pi}(s \mid Y)=\lambda^{0}(Y) \sum_{a \in P^{i, x}(\pi)} \pi(a \mid Y),
\end{aligned}
$$

where the inequality holds since $\alpha^{\hat{\pi}}$ is a BNE. Showing that choosing action $y$ is optimal upon observing message $y$ is similar. It follows that $\pi \in \Pi^{\text {sae }}$.

Proof of Lemma 5.2. If $\lambda^{0}(X) \geq 1 / 2$, then consider $\pi \in \Pi^{\text {sae }}$ with representation $(q, r)$ such that $q_{n}=1$ and $r_{k+1}=1$. Observe that $V^{\pi}\left(\lambda^{0}\right)=1 \geq V^{\hat{\pi}}\left(\lambda^{0}\right)$. Let $\lambda^{0}(X)<$ $1 / 2$. Let $\pi \in \Pi^{\mathrm{a}}$ be defined such that $q_{k-1}=q_{k}=0, q_{k+1}=\hat{q}_{k-1}+\hat{q}_{k}+\hat{q}_{k+1}, q_{\ell}=\hat{q}_{\ell}$ for all $\ell \neq k-1, k, k+1, r_{k}=0, r_{k+1}=\hat{r}_{k}+\hat{r}_{k+1}$, and $r_{\ell}=\hat{r}_{\ell}$ for all $\ell \neq k, k+1$. Clearly, $\pi$ satisfies (16) and (17), so $\pi \in \Pi^{\text {ae }}$. We next show that $\pi$ is straightforward. Since $\hat{\pi}$ is straightforward, it holds that $\lambda^{0}(Y) \sum_{\ell=0}^{n} \frac{\ell}{n} \hat{r}_{\ell} \leq \lambda^{0}(X) \sum_{\ell=0}^{n} \frac{\ell}{n} \hat{q}_{\ell}$. Moreover, as $\alpha^{\hat{\pi}}$ is a $\mathrm{BNE}$, it holds that $\lambda^{0}(Y) \hat{r}_{k} \leq \lambda^{0}(X) \hat{q}_{k}$. Hence,

$\lambda^{0}(Y) \sum_{\ell=0}^{n} \frac{\ell}{n} r_{\ell}=\lambda^{0}(Y)\left(\sum_{\ell=0}^{n} \frac{\ell}{n} \hat{r}_{\ell}+\frac{1}{n} \hat{r}_{k}\right) \leq \lambda^{0}(X)\left(\sum_{\ell=0}^{n} \frac{\ell}{n} \hat{q}_{\ell}+\frac{1}{n} \hat{q}_{k}+\frac{2}{n} \hat{q}_{k-1}\right)=\lambda^{0}(X) \sum_{\ell=0}^{n} \frac{\ell}{n} q_{\ell}$, 
which proves that $\pi \in \Pi^{\text {sae }}$. Finally,

$$
V^{\pi}\left(\lambda^{0}\right)=\lambda^{0}(X) \sum_{\ell \geq k} q_{\ell}+\lambda^{0}(Y) \sum_{\ell \geq k} r_{\ell}=\lambda^{0}(X) \sum_{\ell \geq k} \hat{q}_{\ell}+\lambda^{0}(Y) \sum_{\ell \geq k} \hat{r}_{\ell}=V^{\hat{\pi}}\left(\lambda^{0}\right),
$$

which completes the proof.

Proof of Lemma 5.3. If $\lambda^{0}(X) \geq 1 / 2$, then consider $\pi \in \Pi^{\text {sae }}$ with representation $(q, r)$ such that $q_{n}=1$ and $r_{k+1}=1$. Observe that $V^{\pi}\left(\lambda^{0}\right)=1 \geq V^{\hat{\pi}}\left(\lambda^{0}\right)$. Let $\lambda^{0}(X)<$ $1 / 2$. By Lemma 5.2, we can assume without loss of generality that $\hat{q}_{k}=\hat{r}_{k}=0$. Let $\pi \in \Pi^{\mathrm{a}}$ be defined by $q_{n}=1, q_{\ell}=0$ for all $\ell \neq n, r_{0}=\sum_{\ell=0}^{k-1} \hat{r}_{\ell}, r_{k+1}=\sum_{\ell=k+1}^{n} \hat{r}_{\ell}$ and $r_{\ell}=0$ for all $\ell \neq 0, k+1$. Then $\pi \in \Pi^{\text {sa }}$ since

$$
\begin{aligned}
\lambda^{0}(Y) \pi_{i}(x \mid Y) & =\lambda^{0}(Y) \sum_{\ell=0}^{n} \frac{\ell}{n} r_{\ell}=\lambda^{0}(Y) \frac{k+1}{n} r_{k+1}=\lambda^{0}(Y) \frac{k+1}{n} \sum_{\ell=k+1}^{n} \hat{r}_{\ell} \leq \lambda^{0}(Y) \sum_{\ell=0}^{n} \frac{\ell}{n} \hat{r}_{\ell} \\
& \leq \lambda^{0}(X) \sum_{\ell=0}^{n} \frac{\ell}{n} \hat{q}_{\ell} \leq \lambda^{0}(X)=\lambda^{0}(X) \pi_{i}(x \mid X),
\end{aligned}
$$

where the second inequality holds as $\hat{\pi} \in \Pi^{\text {sae }}$. Since $q_{k-1}=q_{k}=r_{k}=0$, (16) and (17) are satisfied. So, $\pi \in \Pi^{\text {sae }}$. Finally,

$$
V^{\pi}\left(\lambda^{0}\right)=\lambda^{0}(X)+\lambda^{0}(Y) r_{k+1} \geq \lambda^{0}(X) \sum_{\ell=k}^{n} \hat{q}_{\ell}+\lambda^{0}(Y) \sum_{\ell=k+1}^{n} \hat{r}_{\ell}=V^{\hat{\pi}}\left(\lambda^{0}\right)
$$

where the last equality uses that $\hat{r}_{k}=0$.

Proof of Proposition 5.4. If $\lambda^{0}(X) \geq 1 / 2$, then the solution to the linear optimization problem in Proposition 5.4 is given by $\left(r_{0}^{*}, r_{k+1}^{*}\right)=(0,1)$, leading to $V^{* e}\left(\lambda^{0}\right)=1$ as desired. Let $\lambda^{0}(X)<1 / 2$. By Lemmas 5.1, 5.2 and 5.3, if there is an optimal $\hat{\pi} \in \Pi^{\text {sae }}$ then there is $\pi \in \Pi^{\text {sae }}$ with representation $q, r$ such that $q_{k}=1, r_{\ell}=0$ for all $\ell \neq 0, k+1$, and $V^{*}\left(\lambda^{0}\right)=\lambda^{0}(X)+\lambda^{0}(Y) r_{k+1}$. Such $\pi$ is straightforward if and only if

$$
\lambda^{0}(X)=\lambda^{0}(X) \pi_{i}(x \mid X) \geq \lambda^{0}(Y) \pi_{i}(x \mid Y)=\lambda^{0}(Y) \frac{k+1}{n} r_{k+1},
$$

which is equivalent to $(21)$.

Proof of Theorem 5.5. By (21), it holds that

$$
\frac{\lambda^{0}(X)}{\lambda^{0}(Y)} \frac{n}{k+1} \geq r_{k+1}
$$


Since the objective function is increasing in $r_{k+1}$, the optimal value is obtained by choosing

$$
r_{k+1}^{*}= \begin{cases}1 & \text { if } \lambda^{0}(X) \geq \frac{k+1}{n+k+1}, \\ \frac{\lambda^{0}(X)}{\lambda^{0}(Y)} \frac{n}{k+1} & \text { if } \lambda^{0}(X)<\frac{k+1}{n+k+1} .\end{cases}
$$

Hence, if $\lambda^{0}(X) \geq \frac{k+1}{n+k+1}$ then $V^{* e}\left(\lambda^{0}\right)=1$. Otherwise, it holds that

$$
V^{* \mathrm{e}}\left(\lambda^{0}\right)=\lambda^{0}(X)+\lambda^{0}(Y) r_{k+1}=\lambda^{0}(X)+\lambda^{0}(Y) \frac{\lambda^{0}(X)}{\lambda^{0}(Y)} \frac{n}{k+1}=\lambda^{0}(X) \frac{n+k+1}{k+1} .
$$

Proof of Corollary 5.7. (i) These inequalities follow immediately from the simple observation that, for all $k=1, \ldots, n-1$,

$$
2 \leq \frac{n+k+1}{k+1} \leq \frac{n+k}{k} .
$$

(ii) This follows immediately from Proposition 3.6 and Theorems 3.10 and 5.5.

Proof of Corollary 5.8. This follows immediately from the observation that $(n+k+$ $1) /(k+1)$ is decreasing in $k$ and bounded from below by 2 for all $k \leq n-1$.

\section{References}

Alonso, R., Câmara, O., 2016. Persuading voters. American Economic Review 106, 3590-3605.

Alonso, R., Câmara, O., 2018. On the value of persuasion by experts. Journal of Economic Theory 174, 103-123.

Arieli, I., Babichenko, Y., 2019. Private bayesian persuasion. Journal of Economic Theory $182,185-217$.

Battaglini, M., Morton, R.B., Palfrey, T.R., 2010. The swing voter's curse in the laboratory. The Review of Economic Studies 77, 61-89.

Bergemann, D., Morris, S., 2016. Bayes correlated equilibrium and the comparison of information structures in games. Theoretical Economics 11, 487-522.

Buechel, B., Mechtenberg, L., 2019. The swing voter's curse in social networks. Games and Economic Behavior 118, 241-268. 
Chan, J., Gupta, S., Li, F., Wang, Y., 2019. Pivotal persuasion. Journal of Economic Theory 180, 178-202.

Feddersen, T.J., Pesendorfer, W., 1996. The swing voter's curse. The American Economic Review 86, 408-424.

Gentzkow, M., Kamenica, E., 2017. Bayesian persuasion with multiple senders and rich signal spaces. Games and Economic Behavior 104, 411-429.

Grosser, J., Seebauer, M., 2016. The curse of uninformed voting: An experimental study. Games and Economic Behavior 97, 205-226.

Hedlund, J., 2017. Bayesian persuasion by a privately informed sender. Journal of Economic Theory 167, 229-268.

Heese, C., Lauermann, S., 2017. Robust voter persuasion. Working Paper.

Kamenica, E., Gentzkow, M., 2011. Bayesian persuasion. American Economic Review 101, 2590-2615.

Kolotilin, A., Mylovanov, T., Zapechelnyuk, A., Li, M., 2017. Persuasion of a privately informed receiver. Econometrica 85, 1949-1964.

Kosenko, A., 2018. Bayesian persuasion with private information. Working Paper.

Laclau, M., Renou, L., 2016. Public persuasion. Working Paper.

Li, F., Norman, P., 2018. On bayesian persuasion with multiple senders. Economics Letters 170, 66-70.

Mathevet, L., Perego, J., Taneva, I., forthcoming. On information design in games. Journal of Political Economy.

Matyskova, L., 2018. Bayesian persuasion with costly information acquisition Working Paper.

Spenkuch, J.L., 2018. Expressive vs. strategic voters: An empirical assessment. Journal of Public Economics 165, 73-81.

Tsakas, E., Tsakas, N., 2017. Noisy persuasion. Working Paper.

Wang, Y., 2013. Bayesian persuasion with multiple receivers. Working Paper. 